\title{
MöglichKeIten UNd BARRIEREN FÜR Kommunikation und soziale Partizipation Geflüchteter IN ÖSTERREICH IM SPANNUNGSFELD VERSCHIEDENER SozIALITÄTEN
}

Sabine BAUER-Amin, Wien, Institut für Sozialanthropologie, Österreichische Akademie der Wissenschaften

\section{INHALT}

1 Die gegenwärtige politische Debatte zu Integration in Österreich.

2 Kommunikation, soziale Kontakte und deren inhärente Sozialitäten ..................... 177

2a „Sozialität“ statt ,,sozialer Integration“ als Denkkonzept..................................... 177

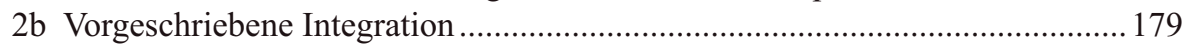

2c Gewünschte Sozialität(en) sozialer Teilhabe ................................................... 181

3 Bestandsaufnahme: Möglichkeiten des sozialen Austausches ................................. 183

3a Kurse im Rahmen staatlicher Integrationsmaßnahmen ..................................... 184

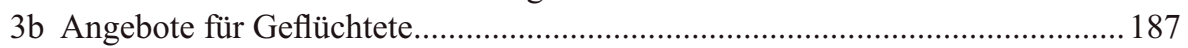

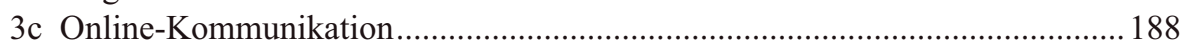

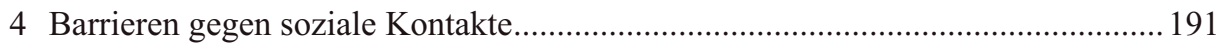

4a Kontaktvermeidung gegenüber der eigenen Sprachgruppe bzw.

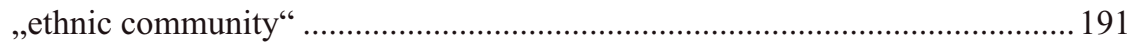

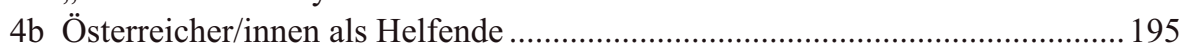

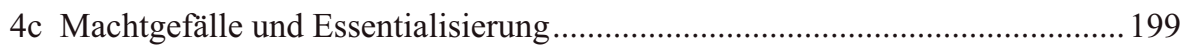

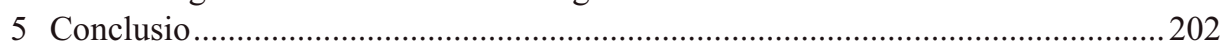

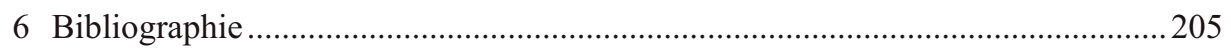




\section{Die gegenwärtige politische Debatte zu Integration in Österreich}

„Parallelgesellschaften verhindern“, so lautet ein Eintrag auf der Internetseite des aktuellen österreichischen Bundeskanzlers Sebastian Kurz im Oktober 2018. Mit Verweis auf diese Agenda wurde im selben Jahr die Schließung mehrerer Moscheen in Österreich und die geplante Auflösung der arabischen Kultusgemeinde gerechtfertigt. Zudem weist der Bundeskanzler in diesem Eintrag gezielt auf die Gefahr durch Geflüchtete aus mehrheitlich muslimischen Ländern hin, die laut seiner Einschätzung die Entstehung oder Verfestigung von parallelen Gesellschaften ${ }^{1}$ begünstigen würden (KuRZ 21/10/2018). Obwohl der Begriff bereits in den 2000er Jahren viel diskutiert, kritisiert und infrage gestellt wurde (siehe Aus Politik und Zeitgeschichte APuZ 2006; Bukow et al. 2007; Gaitanides 2001; Meyer 2002), ${ }^{2}$ erlebt er in der aktuellen Flüchtlings- und damit einhergehend Integrationsdebatte seit 2015 in Österreich neuen Aufwind. Der gegenwärtige öffentliche Diskurs ist geprägt von einem Wiedererstarken der Idee der „Parallelgesellschaften,“ welche ohne oder mit nur wenigen Kontakten zu einer Mehrheitsgesellschaft entstünden (Siehe Diskussion in Der Standard 1/12/2017b). Der Debatte zu Grunde liegt die Befürchtung einer ,,völligen Abkapselung einzelner Bevölkerungsgruppen“ (WolF 2017, p. 5) basierend auf „[p]atriarchale[n] Strukturen, fehlende[n] Sprachkenntnisse[n], mangelnde[r] Arbeitsmarktintegration“ " und „ethnische[r] bzw. religiöse[r] Abschottung“( (Wolf 2017, p. 5) sowie der damit einhergehenden Gefahr für die Demokratie, da die Existenz von „Parallelgesellschaften“ „die soziale Kohäsion und somit das Fundament von demokratisch freiheitlichen Rechtsstaaten gefährdet.“ (MANEA 2017, p. 6).

Das Konzept kam in den Sozialwissenschaften Ende der 1990er Jahre auf (siehe Heitmeyer et al. 1997, p. 192) ${ }^{3}$ und wurde ab den 2000ern konkret für soziale Kollektive verwendet, die ethno-kulturelle bzw. religiös-kulturelle Homogenität sowie einen gewissen Grad an lebensweltlicher, zivilgesellschaftlicher und ökonomischer Segregation aufwiesen, eigene parallele Institutionen ausbildeten und eine formal quasi freiwillige Segregation sowie eine räumliche oder auch sozial-interaktive Segregation mit sich brachten (MeYer 2002, p. 344). „Parallelgesellschaften“ konnten sich jedoch in der wissenschaftlichen Debatte nicht durchsetzen, da eine Differenzierung nach verschiedenen homogenen, parallelen Gesellschaften empirisch nicht haltbar war (siehe HaLM \& SAUER 2006, p.19), eine Ethnisierung sozialer Probleme beinhaltete und Subgruppen etwa innerhalb der Mehrheitsgesellschaft ignorierte. Zudem zeigten sich auch konzep-

\footnotetext{
Für weitere Informationen siehe „Parallelgesellschaften verhindern“ auf der Internetseite von Sebastian Kurz.

Der Terminus „Parallelgesellschaft“ lag bei der von der Gesellschaft für deutsche Sprache (GfdS) initiierten Wahl zum „Wort des Jahres“ 2004 an zweiter Stelle.

Der Begriff der „Parallelgesellschaft“ wird von Heitmeyer erstmals 1996 in einem Interview über die 1997 veröffentlichte Studie zum Fundamentalismus türkischer Jugendlicher in Deutschland verwendet.
} 
tionelle Schwachpunkte. HALM und SAUER kritisieren vor allem, dass in der weitgehend akzeptierten Definition von MEYer (2002) nicht auf die Frage nach der Ursache von Segregation eingegangen wird. MEYER blende soziale, kulturelle und ökonomische Ausgrenzungen aus. Durch seine negative Schuldkonnotation, so Werner ScHIFFAUER weiter, dient es als Kampfbegriff und nicht als sozialwissenschaftlicher Analysebegriff (SCHIFFAUER 2006 [2005], p. 4 zit. nach KARPENSTEIN, J. et al. 2007, p. 18). Dennoch hat sich die Idee in politischen und medialen Debatten hartnäckig gehalten und beeinflusst nach wie vor gegenwärtige Diskussionen im deutschsprachigen Raum (vgl. KAZIM 24/12/2012; StAAs 10/02/2011; KüCüK \& Fischer 02/09/2018; KROHN 24/07/2018). Der Begriff der „Parallelgesellschaften“ ist somit politischer Natur. Im wissenschaftlichen Sinne wird er aus den genannten Gründen aber kaum mehr verwendet.

Politische und mediale Debatten scheinen von diesen sozialwissenschaftlichen Diskursen jedoch weitgehend unberührt. Thesen der Entstehung geschlossener Gesellschaften, der „Segregation“ oder desintegrativer Milieus, die patriarchale Gesellschaftsformen, ,Zwangsheiraten“ oder „Ehrenmorde“ begünstigen würden oder ein gesamtgesellschaftliches Zusammenleben herausfordern würden (siehe hierzu ÖsTERREICHISCHER INTEGRATIONSFONDS 2017), zogen sich in den vergangenen Jahren wie ein roter Faden durch Anti-Europa- und Anti-Einwanderungs-Polemiken bis hin zu Integrationsdebatten auf höchster nationaler politischer Ebene (siehe UlRAm 2009). Die vorhandenen Befürchtungen erhielten neuen Wind in der Debatte um die seit 2015 neu angekommenen Geflüchteten, welche überwiegend aus mehrheitlich muslimischen Ländern stammen, was somit ein demographisches Wachstum der formal muslimischen Bevölkerung bedeutete. Dies wird besonders deutlich an der Debatte um das Tragen von Kopftüchern aus religiösen Gründen ${ }^{4}$ oder Debatten um die Verwendung von Schweinefleisch im in Schulkantinen angebotenen Essen (siehe Diskussion in Der StANDARD 2017a), welche mit enormer emotionaler und politischer Symbolkraft ausgestattet wurden und sich in österreichischen politischen Debatten über mehrere Jahre halten konnten. ${ }^{5}$

Diese obstinaten Diskurse existieren oft im selben Raum mit der Forderung nach „Integration“ der Geflüchteten, um auf diese Weise die Entstehung abgeschotteter, ethnisch homogener Gruppen zu verhindern. Sie bestimmen zu einem hohen Grad politische und mediale gegenwärtige Debatten in Österreich. Denkt man diese Diskurse weiter, so implizieren sie Forderungen nach sozialen Kontakten zu einer österreichischen „Mehrheitsgesellschaft“ und somit die soziale Partizipation und die Kommunikation mit Personen außerhalb der jeweils eigenen ethnischen und sprachlichen Gruppe.

Präventiv wurden von staatlicher Seite unterschiedliche Integrationsmaßnahmen festgelegt, wie etwa der verpflichtende schnelle Spracherwerb oder der Besuch staat-

\footnotetext{
$4 \quad$ Siehe Bundesgesetz über das Verbot der Verhüllung des Gesichts in der Öffentlichkeit (AntiGesichtsverhüllungsgesetz - AGesVG) von 2017. Das Verschleierungsverbot verfolge nach $\S$ 1 ,die Förderung von Integration durch die Stärkung der Teilhabe an der Gesellschaft und die Sicherung des friedlichen Zusammenlebens in Österreich“".

5 Der Begriff „Parallelgesellschaft“ soll im Folgenden nicht als sozialwissenschaftliche Analysekategorie verwendet werden, sondern als Gegenstand der gegenwärtigen politischen Debatte.
} 
lich koordinierter und entwickelter „Werte- und Orientierungskurse“. Der Besuch dieser Kurse und die Absolvierung der anschließenden Modulprüfungen werden als „Integrationspflicht" gesetzlich vorgeschrieben (Integrationsgesetz - IntG 2017, 1 §1). Dennoch zeigt sich in der Realität ein komplexeres Bild von Möglichkeiten sozialer Partizipation, die von vielen verschiedenen Faktoren abhängen und teilweise durch die beschlossenen Maßnahmen eher behindert als gefördert werden. Vor allem der Aufbau sozialer Beziehungen außerhalb der eigenen ethnischen und linguistischen Gruppe wird durch den politischen Diskurs und die daraus resultierenden staatlichen Maßnahmen oftmals erschwert, da selbiger soziale Distanzen aufbaut und Kontaktmöglichkeiten schmälert. Um jedoch in der neuen Gesellschaft anzukommen, ist es notwendig heterogene Netzwerke aufzubauen. Bereits seit Mitte der 1980er Jahre wird in der Migrationsforschung die Bedeutung interethnischer Freundschaften und heterogener sozialer Netzwerke als Basis für die Integration diskutiert (vgl. Esser 1990, p. 185). Damit einher geht oft der Wunsch migrierter Gruppen selbst mehr Kontakte mit der ansässigen Bevölkerung einzugehen (vgl. SChUlERI-HARTIE 1982). In späteren Forschungen wurden heterogene ethnische Beziehungen sogar als Indikator für gelungene Integration benannt (JANSSEN \& Polat 2006: 14; vgl. Esser 2001). Daher liegt es nahe, Möglichkeiten und Barrieren sozialer Partizipation, die in politischen und medialen Debatten eher weniger Aufmerksamkeit erhalten, zu untersuchen und ihre Bedeutung herauszustellen.

Basierend auf einer qualitativen muttersprachlichen Befragung von circa 90 Geflüchteten aus Afghanistan, dem Irak und Syrien wird im Folgenden auf die Erfahrungen und Erwartungen der genannten Gruppe bezüglich sozialen Kontakten und Kommunikation in Österreich näher eingegangen. Für einen Großteil der Befragten ist der (Wieder)aufbau eines sozialen Netzwerkes in Österreich von zentraler Bedeutung. Für eine zielgruppenorientierte Analyse werden im Folgenden arabischsprachige Interviews analysiert und als empirische Beispiele herangezogen. Dabei stehen vor allem die erfahrene und die gewünschte Art sozialer Teilhabe und in weiterer Folge die Diskrepanz zum vorgeschriebenen Verständnis von Integration im Vordergrund.

Im Folgenden wird zunächst auf die Thematik der Sozialität ${ }^{6}$ eingegangen, sodann verschiedene arabische Taxonomien und daran geknüpfte Erwartungen von Sozialität vorgestellt sowie eine Bestandsaufnahme möglicher Kommunikationsmodi und Kommunikationsbarrieren vorgenommen. Dabei sollen insbesondere emergente Machtstrukturen und Ausgrenzungsmechanismen angesprochen werden, die das Knüpfen sozialer Kontakte erschweren. ${ }^{7}$

\footnotetext{
Sozialität soll hier als die Qualität sozialen Verhaltens sowie als eine Tendenz, soziale Beziehungen und Gruppen zu bilden, welche sich reproduzierende Reziprozität erlauben, verstanden werden.

Die folgenden empirischen Daten entstammen dem durch den Innovationsfonds der ÖAW geförderten Projekt „Loslassen - durchstehen - ankommen: Eine transdisziplinäre Studie zur rezenten Situation Geflüchteter in Österreich (LODA)“ des Instituts für Sozialanthropologie, des Instituts für Stadt- und Regionalforschung (beide ÖAW) und des Instituts für Kultur- und Sozialanthropologie (Universität Wien).
} 


\section{Kommunikation, soziale Kontakte und deren inhärente Sozialitäten}

Die beschriebenen Möglichkeiten soziale Kontakte aufzubauen und die Hürden und Barrieren, die damit einhergehen, zeigen, dass es nicht notwendigerweise rein quantitativ an Möglichkeiten zum Austausch mangelt, wenngleich diese in manchen Fällen sehr beschränkt sein können. Es sind durchaus Gelegenheiten des (Wieder)aufbaus sozialer Netze vorhanden. Die Herausforderung besteht jedoch in der Gestaltung der Qualität dieser Kontakte, die in ihrer erlebten Sozialität oft nicht den Vorstellungen und Bedürfnissen der Betroffenen entsprechen. So lassen sich die Kontakte hinsichtlich vorgeschriebener, tatsächlicher und gewünschter Art von Kommunikation und sozialer Partizipation unterscheiden.

\section{$2 a$ „Sozialität“ statt „sozialer Integration“ als Denkkonzept}

In der sozialwissenschaftlichen Literatur wird darauf verwiesen, dass Geflüchtete vor allem in der transitorischen Phase, aber auch darüber hinaus, eine Reihe an Prä- und Postmigrationsstressoren ausgesetzt sind ${ }^{8}$ (siehe KHAwAJA et al. 2008; ONYUT et al. 2009, Sinnerbrik, et al. 1997; SANGalang et al. 2018). Obwohl die Verarbeitung des Erlebten selbst eine enorme Herausforderung sein kann, tritt auch nach der Ankunft eine Reihe an weiteren Stressfaktoren hinzu. Viele Geflüchtete kommen im neuen Land mit begrenztem Wissen über die neue Umgebung und keinen oder eingeschränkten Kenntnissen der Landessprache an (KHAwAJA et al. 2008, p. 503). Hinzu gesellen sich Herausforderungen beim Erlernen der neuen Sprache, beim Zurechtfinden in der neuen Umgebung, die Notwendigkeit des (Wieder-)aufbaus eines sozialen Netzes, das Einfinden in neue soziale Rollen, die Verpflichtung zu Geldsendungen an die Familie etc. Man spricht hier generell vom Postmigrationsstress. Für viele Geflüchtete akkumulieren sich noch weitere Stressoren. Sie haben vor und während der Flucht Haft, Hunger, Folter, Tod von Freunden oder Familienmitgliedern und Gewalt miterlebt (ONYUT et al. 2009, SinNERBRIK et al. 1997). Zu diesen Erfahrungen kommen des Weiteren ihre finanziell prekäre Situation in Österreich, die Sorge um Angehörige im Heimatland, das Auseinanderreißen der Familie, Trauer und Verlust sowie die Ungewissheit, was Aufenthalt und Zukunftsplanung betrifft, hinzu. Wie gut man mit diesen Faktoren zurechtkommt, hängt unter anderem von der Verfügbarkeit von Ressourcen ab, etwa dem Vorhandensein der Kernfamilie, Sprachkenntnissen und Bildung, dem Gefühl der relativen Sicherheit in Österreich oder der Verfügbarkeit tragender sozialer Beziehungen vor Ort. Unter den genannten Voraussetzungen ist es oft nicht leicht, den staatlich vorgeschriebenen Integrationsanforderungen nachzukommen. Soziale Beziehungen im neuen Residenzland aufzubauen ist somit ein wesentlicher Aspekt, um die genannten Umstände besser bewältigen zu können. Zudem repräsentieren diese Multiplikatoren, dass sie gerade zum

Obwohl es hier um Geflüchtete geht, lautet der Fachterminus Postmigrationsstress. 
Aufbau weiterer Ressourcen wichtig und gewünscht sind und etwa beim Spracherwerb, aber auch beim Zurechtfinden auf dem Arbeitsmarkt förderlich sein können. Soziale Bindungen entstehen in Österreich oft entlang des Integrationsprozesses. Jedoch sind nicht alle sozialen Beziehungen einander gleichzusetzen (Mikal \& Woodfield 2015, p. 1320), denn diese können sich nicht nur hinsichtlich ihrer Häufigkeit, sondern auch bezüglich der Art der Sozialität unterscheiden.

In der Diskussion um verschiedene Aspekte der Integration wird oft mit Bezug auf David Lockwood (1964) und Hartmut Esser (2001) zwischen System- und Sozialintegration unterschieden. Während Systemintegration laut dieser Unterscheidung die Gesellschaft als das Bezugssystem sieht und auf die Integration in die einzelnen Teile des Systems fokussiert, sieht Sozialintegration die Akteure der Bevölkerung als Bezugssystem. Sozialintegration versteht somit ,die Integration der Akteure (bzw. der von ihnen gebildeten Gruppen) ,in“ das System hinein“ (Esser 2011, p. 3). Auch andere Unterscheidungen sind vorstellbar, wie sie etwa von KARPENSTEIN et al. (2007) vorgenommen werden. Letztere unterscheiden zwischen systemischer, soziokultureller und kultureller Integration. Erstgenannte Integration versteht die Teilhabe an Teilsystemen der Gesellschaft, wie Bildung, Politik, Recht oder Wirtschaft. Soziokulturelle Integration meint die wahrgenommene Distanz oder Nähe und damit die Identifikation mit der Mehrheitsgesellschaft und inkludiert sowohl das Freizeitverhalten wie auch das Knüpfen sozialer Beziehungen. Kulturelle Integration meint schließlich das Erlernen von Sprache etc. (ebenda, p. 18). Allerdings betonen sozialwissenschaftliche Konzepte der Integration seit längerer Zeit, dass sich Aspekte des sozialen Zusammenlebens nicht trennen lassen von strukturellen Aspekten. Daher besteht die Forderung, eine ganzheitliche Betrachtungsweise einzunehmen und deren wechselseitige Bedingtheit zu berücksichtigen. (vgl. Esser 2001, pp. 1,4; Ager \& Strang 2008, pp. 169-171). So ist zwar eine Systemintegration ohne Sozialintegration möglich, jedoch bedingen einander beide vielfach, wie im Folgenden dargestellt wird. Basierend auf dieser wechselseitigen Beziehung ist es sinnvoll, soziale Partizipation nicht durch unterschiedliche Teilbereiche von „Integration“" strukturiert zu denken, sondern entlang der Qualitäten sozialer Teilhabe.

Während das Konzept der „Integration“ einen Prozess zur Schaffung sozialer Teilhabe und Eingliederung beschreibt, erklärt es nicht, welche Art der Teilhabe hergestellt wird und wie sich Beziehungen entlang von Integrationsprozessen gestalten. Soziale Interaktionen und Beziehungen zivilgesellschaftlicher und individueller Natur können kaum gesetzlich in Top-down-Regelungen festgeschrieben werden. Im neuen Integrationsgesetz findet man daher einen Fokus auf struktureller Integration, wie Arbeitsmarktintegration und das Erlernen der Sprache. Dennoch wird betont, dass Integration auf „persönlicher Interaktion beruht“ (IntG 2017, (1) § 1). Die entstehenden Kontakte, die zu einem großen Teil aus diesen staatlichen Maßnahmen hervorgehen, schaffen jedoch Abhängigkeiten sozialer Steuerung durch staatliche Institutionen, Isolation entlang linguistischer Gruppe, Zugehörigkeiten entsprechend gesetzlich geschaffener Kategorien und asymmetrische Rollenzuschreibungen zwischen Österreicher/inne/n und Geflüchte- 
ten. Die somit entstehenden Kontakte beinhalten unterschiedliche Arten von Sozialität. Die Entwicklung dieser Sozialitäten können nicht nur von tatsächlichen Verhältnissen angetrieben werden, sondern auch Wunschvorstellungen für zukünftiges Miteinander oder Alternativen zum jetzigen Zustand sein. Allerdings warnt Christina Toren davor, Sozialität als festgeschriebene analytische Kategorie zu verwenden (2013, p. 46). Sozialität darf nicht als statische Konstante der Zugehörigkeit oder Nichtzugehörigkeit verstanden werden. Nicholas Long und Henrietta Moore (2013) weisen darauf hin, dass auch die Kapazität über mögliche, zukünftige oder alternative Arten der Sozialität nachzudenken und bestehende Formen zu ändern Teil der Analyse sein müsse (ebenda, p. 1), da selbige genauso Einflussfaktoren auf erlebte Sozialität sind, wie Antriebsfaktoren diese zu ändern. Sozialität soll daher als ein prozessuales Produkt aus sozialen Beziehungen und Interaktionen verstanden werden (ebenda, p. 2) und ist dadurch eng verbunden mit dem Konzept der sozialen Integration, wobei es jedoch mehr den Fokus auf die Arten der Interaktionen und die sich daraus ergebenden Zugehörigkeiten legt. Die Autor/inn/en proklamieren, dass soziale Akteur/inn/e/n stets ein Interesse an Beziehungsdynamiken haben, welches sowohl durch den Wunsch als auch den Widerwillen, diese in neue Richtungen zu beeinflussen, ausgedrückt werden (ebenda, p.3). Daher ist Sozialität eng mit Fragen nach Wunsch, Motivationen und Ethik verbunden und legt den Fokus der Untersuchung sozialer Partizipation auf intrinsische Motive von Akteur/inn/ en sowie deren Erleben von Barrieren, die die gewünschte Sozialität erschweren. Die Erfahrungen und Wünsche Geflüchteter hinsichtlich sozialer Beziehungen und Kommunikation unter dem Gesichtspunkt von Sozialität zu beleuchten ermöglicht daher eine stärkere Fokussierung auf die Sicht der Betroffenen selbst und rückt deren agency vor dem Hintergrund institutioneller Steuerungen in den Vordergrund.

\section{$2 b$ Vorgeschriebene Integration}

Das aktuelle österreichische Integrationsgesetz sieht vor, dass Personen möglichst schnell Deutsch lernen, um zügig in den Arbeitsmarkt integriert werden zu können. Damit soll verhindert werden, dass es zur Entstehung der eingangs beschriebenen „Parallelgesellschaften“ kommt. Daher ist Integration im Gesetz auch als ,gesamtgesellschaftlicher Prozess, dessen Gelingen von der Mitwirkung aller in Österreich lebenden Menschen abhängt und auf persönlicher Interaktion beruht" festgeschrieben (IntG $2017 \S 2$ (1)). Um dies zu gewährleisten, wurde eine Integrationsvereinbarung ausgehandelt. In dieser verpflichten sich Geflüchtete zu Integrationsleistungen, deren Erfüllung in Form von Integrationsprüfungen kontrolliert wird. Personen, die diese Leistungen nicht erfüllen können und beispielsweise keine entsprechenden Sprachkenntnisse vorweisen können, erhalten dementsprechend weniger Sozialleistungen oder es werden ihnen andere, zumeist finanzielle und gesetzlich festgeschriebene Sanktionen auferlegt.

Dies führte in den häufig frequentierten Facebookgruppen Geflüchteter zu beträchtlicher Verunsicherung und löste zusätzlichen Druck aus. Die Maßnahmen und staatlich 
vorgeschriebenen Initiativen wurden in den arabisch-sprachigen Interviews häufig als indimāj ${ }^{9}$ beschrieben und automatisiert. Dies leitet sich ab von Arabisch damaja, welches als hineinkommen, einfügen oder absorbiert werden übersetzt werden kann. In den Interviews wurde der Terminus meist in Verbindung mit den gesetzlich vorgeschriebenen Integrationskursen benutzt, wie aus folgenden Beispielen ersichtlich wird.

Auf die Frage, was ein 53-jähriger Syrer unter Integration (indimāj) verstehe, antwortete seine Tochter etwa sofort: „Den Kurs hat er schon gemacht.“ Auf weitere Nachfrage, erklärte er: ,Integration heißt Teil des Arbeitsmarktes zu sein. Integration ist Sprache und Arbeit. Ich bin alt, aber ich lerne." Die Antwort des Mannes entsprach den vorgeschriebenen Maßnahmen und dem Ziel des Integrationsgesetzes, bezog sich aber nicht auf den Kontakt mit der österreichischen Gesellschaft, obwohl er in anderen Teilen des Interviews öfter betonte, wie wichtig es ihm sei, sein soziales Leben in Österreich zu verbessern. Die defensive Rechtfertigung, dass er trotz seines Alters lerne, zeigte, dass er Integration hauptsächlich als staatlichen Druck und eine vorgeschriebene „Integrationspflicht" erlebte.

Integration als hauptsächlicher Formalakt, der als Top-down-Verpflichtung lediglich die staatlichen Maßnahmen betrifft, war ein in den Interviews weitverbreitetes Verständnis. So antwortete etwa ein 44-jähriger syrischer Asylwerber auf die Frage, ob er schon Kurse des ÖIF besucht habe: „Integrationskurse des ÖIF? Integration? Das mache ich erst, nachdem ich den Asylbescheid erhalten habe. Ich mache dann die Integrationskurse und lerne die Sprache, damit ich mich in das Land hier integriere. Das ist notwendig. Wenn man Teil der Gesellschaft sein will, muss man die Sprache lernen.“ Hierbei wird deutlich, dass sich der Mann eines linear vorgegebenen Integrationspfades bewusst war, in dessen Verlauf er sich sozusagen zur Leistung einer Bringschuld verpflichtet sah. Diese beinhaltet eine Pflicht des Geflüchteten gegenüber Österreich. Diese regelt somit zwar das Verhältnis von Staat und Individuum, nicht aber die Anknüpfung und den Ausbau sozialer Kontakte.

Manchmal wurde indimāj auch als eine Fähigkeit betrachtet, die man erlernt, wie etwa die fremde Sprache. Ein 34-Jähriger Familienvater beschrieb dies folgendermaßen: „Ich habe in Russland studiert. Ich war zwei Jahre in Russland. Ich habe dort eine gute Integration (indimāj) gemacht, weißt du. Ich habe echt schnell gelernt. Aber jetzt habe ich fast alles vergessen. Als ich zurück nach Syrien ging, habe ich meine Integration (indimāj) aus Russland vergessen.“

Die staatlichen Maßnahmen zur Förderung von Integration erzeugen daher eine Beziehung zwischen Geflüchteten und dem Staat basierend auf rechtlichen Verpflichtungen und zugleich einen enormen Leistungsdruck. Dem Ziel, damit soziale Segregationstendenzen zu verhindern, können diese Maßnahmen jedoch nicht gerecht werden, da sie keine Möglichkeiten schaffen, feste und stabile Bindungen zu Personen außerhalb der Sprachcommunity zu etablieren, stattdessen Druck aufbauen und Strukturen fördern,

Eingliederung, Verschmelzung, Fusionierung, Assimilation (Übersetzung nach WeHR 1985, p. 404). 
die zu Isolation und Vereinsamung führen können, wie im Folgekapitel beschrieben wird. Wenngleich das Ziel der Integrationsmaßnahmen die „Teilhabe am gesellschaftlichen, wirtschaftlichen und kulturellen Leben in Österreich“" (IntG 2017 §2 (2)) ist, so werden staatliche Integrationsmaßnahmen oft als Verpflichtung, Formalakt oder gar als eine Fertigkeit gesehen, weniger aber als eine Form sozialer Teilhabe.

\section{$2 c$ Gewünschte Sozialität(en) sozialer Teilhabe}

Wenn befragte Personen beschrieben, wie sie ihr Zusammenleben mit hier Ansässigen gerne gestalten würden, so benutzten sie zumeist arabische Termini, die eine Vermischung oder Vermengung beschrieben. Zumeist wurde das Wort mazaja verwendet. Dies wird von Hans WEHR als vermischen oder vermengen übersetzt (ebenda 1985, p. 1203). Ebenso wurde halața zur Beschreibung gewünschter Kontakte benutzt, was als mischen, zugesellen oder (durcheinander) mengen übersetzt wird. Auch wașala kam als Charakterisierung gewünschter Sozialität vor, welches verbinden, vereinigen, zusammenschließen bedeutet. Dies ging oft mit dem Wunsch einher, Kontakte vor allem zu den österreichischen Nachbar/inne/n aufzubauen und einander gegenseitig z.B. auf eine Tasse Kaffee einzuladen oder anzurufen. Der Wunsch nach dieser Art von Sozialität war durchaus gegeben, allerdings war dieser nicht zwangsweise deckungsgleich mit dem Verständnis von staatlicher Integration, welche weniger auf zwischenmenschliche Beziehungen setzt, als vordergründig vermuten lässt und die Arbeitsmarktintegration in den Vordergrund stellt. Die gewünschte Sozialität sozialer Teilhabe basiert auf einem Verständnis der „Vermischung“, wie es dem arabischen Sprachgebrauch zu Grunde liegt.

Neben den staatlichen Verpflichtungen thematisierten Geflüchtete das Verhältnis $\mathrm{zu}$ anderen in Österreich Lebenden, also hauptsächlich zu Österreicher/inne/n und anderen Geflüchteten. Wie bereits beschrieben, stand hier nicht das offiziell vorgegebene Verständnis von Integration im Vordergrund, sondern ein fast wörtlich zu übersetzendes „Unter-die-Leute-mischen“ oder „Zusammenschließen“. Viele Personen wünschten sich ein dichteres und erfüllenderes soziales Netzwerk, welches auch in der Literatur als besonders wichtig für die Bewältigung von Postmigrationsstress und Integrationsbarrieren genannt wird (MIKAL \& WoODFIELD 2005, p. 1319). In den folgenden Beispielen werden nun die gewünschten Qualitäten sozialer Kontakte beschrieben.

In vielen Interviews wurde ein Verhältnis zu Österreicher/inne/n gewünscht, welches auf Reziprozität beruht und in welchem Obligationen und Entgegenkommen gleichermaßen wahrgenommen werden. Dies zeigt sich zum einen an der großen Anzahl der Teilnehmenden, die selbst in irgendeiner Form Freiwilligenarbeit geleistet haben und zum anderen am oftmaligen Wunsch „richtige“ Freunde in Österreich zu haben, einander gegenseitig zu besuchen und im Alltag zu unterstützen, wie etwa auf die Kinder der Nachbar/inne/n aufzupassen. Dadurch soll, wie Long und Moore beschreiben, eine Art der Sozialität entstehen, welche die beiden Autor/inn/en folgendermaßen beschrei- 
ben: ,constituted by and through subjects actively engaged in a collective being-in-theworld." (Long \& Moore 2013, p. 14). So beschreibt ein 27-jähriger Kurde, dass er sich nach Beziehungen sehne, die auf gleicher Augenhöhe stattfinden. Er erklärt: „Wenn ich genauso gesehen werde wie jeder andere, dann fühle ich mich gut. Und wenn ich verstanden werde mit dem, was ich sage."

Vor allem die Wichtigkeit eines engen Verhältnisses zu Nachbar/inne/n wurde wiederholt vorgebracht, wie etwa in dem Beispiel einer 33-jährigen syrischen Mutter: „Alle Leute [in dem Dorf, in welchem sie in der Grundversorgung war] waren sehr hilfreich. Auch unsere Nachbarn waren sehr nett. Du weißt ja, in der arabischen Welt zählen die Nachbarn wirklich viel. Im Dorf war das auch so. Hier in der Stadt ist es anders. Dort haben unsere Nachbarn immer nach uns gefragt und uns besucht und uns auch zu sich eingeladen." Wie in der Interviewpassage schon angedeutet, wurde diese Art der Sozialität zwischen Nachbar/inne/n zwar oft in der Anfangsphase auf dem Land erlebt, aber nach dem Umzug nach Wien vermisst und wieder herbei gesehnt. ${ }^{10}$

Gerade das Bedürfnis nach einem Mehr an Miteinander mit Österreicher/inne/n wurde in den Interviews immer wieder thematisiert. Dabei standen für die Geflüchteten aber nicht soziale Aktivitäten wie Kurse oder Ausflüge im Vordergrund, sondern das tägliche Miteinander. Der fehlende Umgang wurde etwa von einem 27-jährigen Iraker thematisiert. Er hatte einen der wenigen privaten Plätze in einer Wohngemeinschaft mit Österreicher/inne/n ergattert und war sichtlich darüber enttäuscht, dass dies nicht zwangsweise zu einem Gemeinschaftsgefühl geführt hat, wie er es sich gewünscht hätte. „Ich habe mit Österreicher/inne/n zusammengelebt. Aber ich habe sie kaum gesehen. Ich weiß gar nicht, wo sie waren. Sie sind nur am Abend nach Hause gekommen und haben sich schnell Nudeln gemacht. [...] Jeder hat sein eigenes Essen und isst alleine. Ich hasse das. Ich kann doch nicht alleine essen. Wir haben kaum miteinander gesprochen." Das Nebeneinander statt eines Miteinanders war für den jungen Mann so unerträglich, dass er schließlich auszog. Wie auch in diesem Beispiel angesprochen, lag für viele Befragte das Bedürfnis nach Kontakt nicht in der Quantität der Kontakte zu Österreicher/inne/n, sondern in der qualitativen Gemeinschaft in alltäglichen Situation.

Viele Befragte meinten, dass Geflüchtete nicht nur die Integrationsmaßnahmen erfüllen wollten, um somit offiziell Teil der Gesellschaft zu werden. Sie wünschten sich auch sozial in partizipatorische und reziproke Beziehungen zur ansässigen Bevölkerung eingebunden zu sein, welche weder auf Flüchtlingshilfe noch auf vorgeschriebenen Maßnahmen basierten. Damit wird nun auch verständlich, wieso Personen für diese Art der Sozialität die Begriffe mazaja, halața oder wașala verwendeten und nicht damaja. Der Unterschied dieser arabischen Konzepte ist, dass damaja von einem passiveren Kleineren ausgeht, welches in das Größere eingegliedert wird. Dahingegen legt mazaja den Fokus auf das aktive Vermengen zu gleichen Teilen. Während damaja also einen

\footnotetext{
$10 \quad$ Vgl. hierzu auch die in den Sozialwissenschaften bereits seit langem diskutierte Frage nach der Integrationskraft der Städte (z.B. HEITMEYER et al. 1998).
} 
Top-down-Prozess beschreibt, gibt mazaja einen partizipatorischen Prozess vor und stützt sich auf eine gewünschte Sozialität, bei welcher die Handlungsmacht (agency) der Akteur/inn/e/n im Vordergrund steht. Die in den Interviews beschriebenen Arten gewünschter Sozialität stehen daher im Gegensatz zu der medial und politisch geschürten Angst vor einer „Abschottung“ der Geflüchteten und der Entstehung einer „Parallelgesellschaft". Allerdings sind unterschiedliche strukturelle Hindernisse vorhanden, welche diese Art der gewünschten partizipatorischen Sozialität erschweren.

\section{Bestandsaufnahme: Möglichkeiten des sozialen Austausches}

Viele Personen, die nach Österreich gekommen waren, haben den Großteil ihrer sozialen Netze verloren. Oft sind wichtige Personen verstorben, zurückgeblieben oder über mehrere Länder verteilt, weshalb eine der schwierigsten Aufgaben für Geflüchtete in Österreich darin besteht, neue soziale Kontakte aufzubauen und ein funktionierendes Netzwerk zu etablieren. Ohne dieses Netzwerk ist es oft sehr schwer mit dem Erlebten und dem gegenwärtigen Postmigrationsstress zurechtzukommen.

Ein 29-jähriger Syrer beschreibt beispielsweise, dass er über keine tragfähigen sozialen Beziehungen in Österreich verfüge. Wenn er Probleme habe, so habe er niemanden, an den er sich wenden könne. „Ich denke über meine Probleme selber nach. Alleine. Aber ich hätte gerne einen Freund oder eine Freundin, mit dem ich sprechen könnte. Ich wünschte, ich hätte hier jemanden. Ich hatte einen besten Freund in Syrien, aber er ist im Krieg umgekommen."

Soziale Teilhabe wurde von Geflüchteten im Rahmen ihrer Kontakte mit der eigenen Sprachcommunity, aber auch darüber hinaus durchaus erlebt. Allerdings beinhalteten sie Formen der Sozialität, die im Widerspruch zur gewünschten Sozialität standen. Sie waren nicht auf Gegenseitigkeit oder persönlicher Interaktion aufgebaut. In den Berichten der Geflüchteten spiegelte sich ein kompliziertes Bild wider, was die Art der sozialen Teilhabe und Kommunikation betrifft. Die meisten Kontakte wurden demnach mit anderen Kursteilnehmer/inne/n geschlossen, welche vorwiegend einen ähnlichen sozialen und herkunftsmäßigen Hintergrund aufweisen, oder mit Personen, die in der Flüchtlingshilfe aktiv waren.

Gerade in der Anfangsphase beschrieben viele Studienteilnehmer/innen, dass sie vermehrt Kontakt zu Österreicher/inne/n hatten. Dabei bezogen sie sich vor allem auf Polizei, Behörden, Hilfsorganisationen, aber auch auf Ehrenamtliche. Dieser anfangs intensivere Kontakt flachte aber bei vielen Befragten ab, sobald sie eine Aufenthaltserlaubnis erhielten und damit weder regelmäßig mit Behörden noch mit Hilfsorganisationen zu tun hatten. 
Nach der ersten Phase des Ankommens und Abwartens wurden häufig Kontakte zu einzelnen Personen, häufig Ehrenamtliche, genannt, zu welchen (noch) Kontakt bestand. Mit dem Erhalt des Aufenthaltstitels verließ ein Großteil der Geflüchteten die oftmals kleineren Ortschaften, in welchen sie während der Grundversorgung untergebracht waren, was auch zu einem Verlust der anfangs aufgebauten Kontakte führte. Im weiteren Verlauf wurden Möglichkeiten sozialer Partizipation vor allem in Sprachkursen oder (verpflichtenden) Veranstaltungen und Projekten im Rahmen von Integrationsmaßnahmen und der Flüchtlingshilfe genannt. Aber auch die Teilnahme in Onlineforen und Gruppen in sozialen Medien war ein oft genannter Ort des Austauschs. Hier wurden zum einen Kontakte zur ansässigen Bevölkerung (mit und ohne Migrationsbiographie) aufgebaut, aber auch Freundschaften und Bekanntschaften mit anderen Geflüchteten geschlossen und Kontakte zur Familie aufrechterhalten. Unterstützender Austausch kann dabei helfen, bestehende Unsicherheiten zu bewältigen und ein gewisses Maß an Kontrolle zurückzuerhalten (Rains \& Young 2009, pp. 311 f.). Soziale Medien schienen hierbei eine besondere Bedeutung einzunehmen, da sie es ermöglichten sowohl sprachliche als auch geographische Limitationen der Kommunikation zu umgehen.

\section{$3 a \quad$ Kurse im Rahmen staatlicher Integrationsmaßnahmen}

Für viele Befragte waren die Sprachbarrieren eines der zentralen Hindernisse, um Kontakte in Österreich außerhalb der eigenen Sprachgruppe zu schließen. Oft wurde daher die Überwindung dieser Schwelle als wichtigstes Ziel und erster Schritt der Integration in Österreich beschrieben. Allerdings wurde mehrfach Kritik an der strukturellen Zusammensetzung der Sprachkurse geübt.

Viele der Kurse, an denen die Befragten partizipierten, waren nach Sprachgruppen organisiert. Dies betrifft nicht nur Deutschkurse, sondern auch die Wertekurse oder weitere Maßnahmen, wie etwa die Kompetenzchecks. ${ }^{11}$ Die meisten sozialen Kontakte der Geflüchteten ergaben sich während dieser Kurse. Gerade zu Beginn einer solchen Kursveranstaltung sowie des Aufenthaltes generell sind Personen, welche dieselbe Sprache sprechen und zu welchen es womöglich bereits über Bekannte Beziehungen gibt, wichtige Bezugspersonen. Es existiert somit eine gemeinsame Sprache, man kann wichtige Informationen austauschen und sich auf einen kollektiven Erfahrungsschatz stützen (vgl. Forschungsbereich beim SachverständigenRat Deutscher Stiftungen für IntGration und Migration 2017, p. 73). Zudem kann die Teilnahme an derartigen Netzwerken dem erhöhten Postmigrationsstress entgegenwirken (MIKAL \& WoOdFIELD 2005, p. 1319).

Allerdings hatte diese Vorauswahl nach Sprachgruppen auch negative Folgen. So fand kein Austausch mit Personen einer anderen Sprachgruppe statt, weshalb weder die Notwendigkeit bestand während der Kurse auf Deutsch zu kommunizieren, noch die Möglichkeit überhaupt Kontakte über die eigene Gruppe hinausgehend zu knüp-

11 Siehe den Artikel von Gatterbauer in diesem Band. 
fen. Der/die einzige verbleibende Kontaktpartner/in war somit die Lehrperson. Dieses Faktum wurde in vielen der Interviews kritisch hinterfragt, wie das folgende Beispiel zeigt:

„Interviewer-I: Was sind die typischen Herausforderungen, die Sie im täglichen Leben erleben?

Respondent-R: Die Sprache.

I: Wie zeigt sich das?

Freundin des

Respondenten-G: Er hat wenig Kontakt zu Österreicher/inne/n.

R: Ja, ich habe die Sprache nur im Unterricht gelernt. Daher kann ich nicht so sprechen, wie ich möchte. In Situationen im richtigen Leben [außerhalb des Unterrichtsraumes] kann ich nicht sprechen. Und viele Dinge, die ich gelernt habe, habe ich bereits vergessen. Wenn ich eine gute Stelle haben möchte, muss ich aber gut sprechen. [...]

R: [In den Sprachkursen] spricht jeder nur Arabisch. Das ist auch ein Problem. Durch diese Kurse sollten wir eigentlich Deutsch reden. Aber wenn alle Personen aus Syrien und dem Irak kommen, reden wir natürlich auf Arabisch miteinander.

G: Auf Arabisch ist es viel leichter Kontakte miteinander zu knüpfen. Du kommst aus Syrien und Du kommst aus dem Irak. Warum sollte ich mit Dir Deutsch sprechen? Deshalb spricht man Arabisch.

R: Ja, wenn es gemischte Gruppen wären und zum Beispiel jemand aus Afghanistan oder Polen oder Serbien dabei wäre, dann müssten wir Deutsch miteinander sprechen. " (Iraker, 25 Jahre, und Syrerin, 23 Jahre)

Ähnlich äußerte sich auch ein 27-jähriger Syrer aus Dera'a. ,Das Schlechte an den Kursen ist, wenn ich das so sagen kann, dass wir alle Syrer sind. Das ist, wo das Problem liegt. Wir sind alle Araber, Syrer und Iraker, in den Kursen. Unser Lehrer war Italiener. Er hat uns beobachtet und oft lustige Geschichten erzählt. Einmal wurde er sehr wütend, weil wir alle auf Arabisch miteinander gesprochen haben. Er wurde sehr nervös und hat dann Italienisch mit uns gesprochen. [...] Diese Gruppe dort, alle Araber, wir sprechen alle Arabisch miteinander. Dadurch werden wir nie unsere Sprachkenntnisse verbessern. Aber wenn man aus unterschiedlichen Ländern kommt, wird man eine andere Sprache sprechen müssen. Wenn du vier Syrer hast, wir sind ja die meisten unter den Flüchtlingen, und andere Ausländer aus Asien oder anderen Teilen Europas, wäre das viel besser. Es wäre viel nützlicher. Wenn wir dort alle Araber sind, sprechen wir nur alle in derselben Sprache miteinander.“

Auch wurden die Kurse je nach freien Plätzen zugeteilt. Es gab auch separate Kurse für Männer und Frauen, wobei die Teilung für viele Teilnehmer/innen eher auf einem hartnäckigen Vorurteil als auf dem Bestreben, die Kurse effizienter zu gestalten, 
beruhte. Stattdessen schlugen Respondent/inn/en vor, die Kurse eher nach Alter oder Vorbildung zu organisieren, um auf diese Weise sicherzustellen, dass alle Beteiligten die Kurse möglichst effektiv nutzen konnten. Eine 48-jährige Syrerin, die in Syrien nur bis zu ihrem 12. Lebensjahr die Schule besucht hatte und in Österreich erst das lateinische Alphabet lernen musste, beschrieb ihre erheblichen Schwierigkeiten, dem Tempo der Kurse folgen zu können. Sie erklärte: „Tick, tick, tick, lesen, schnell, schnell. ${ }^{12}$ So verbringen wir jeden Tag. Alles, was wir nicht schaffen, müssen wir zu Hause als Hausübung machen. [...] Schau, er sagt, das ist eine Gabel, das ist das und das ist das. Natürlich können wir uns das nicht merken. Es gibt Leute im Kurs, die nicht lesen können. Und sie wissen auch nicht, was das und das ist. Er [der Lehrer] muss Geduld mit uns haben. Er sagt uns, macht das und lest das und geht dorthin. Er weiß gar nicht, was wir denken. [...] Zum Beispiel kenne ich jetzt die Zahlen. Aber es hat sehr lange für mich gedauert. Aber dann gibt es auch Leute, die überhaupt nie gelernt haben zu schreiben. Und sie können immer noch keine Zahlen schreiben. [...] Er sollte uns doch helfen. Aber alles geht viel zu schnell, weißt du.“"

Nicht nur der Bildungshintergrund, sondern auch das Alter spielt eine wesentliche Rolle und wurde häufig als Parameter zur Einteilung der Kursbesucher/innen vorgeschlagen. Dies beschreibt ein 30-jähriger ehemaliger Sprachlehrer aus dem kurdischen Teil Syriens folgendermaßen: „Da gibt es unterschiedliche Altersgruppen in den Kursen. Da sind ältere Leute und sehr junge Leute. Fast schon Minderjährige und Rentner! Dieser Prozess ist sehr schlecht, um eine Sprache zu erlernen. Da gäbe es auch andere Systeme. [...] Es geht um verschiedene Kriterien. Es geht darum, ob sie hohe Kriterien haben. In Österreich haben sie niedrige Kriterien für die Lehrer. Das AMS entscheidet mit sehr wenig Geld: die billigsten Lehrer.“

Infolge der Organisation der Kurse nach Herkunftssprachgruppen in Kombination mit einer erheblichen Heterogenität hinsichtlich des Alters und der Vorbildung innerhalb der Sprachgruppen werden nicht nur existente Sprachbarrieren weniger rasch abgebaut, sondern soziale Situationen geschaffen, die es den Teilnehmer/inne/n weitgehend verunmöglichen, Kontakte über die Sprachgruppen hinweg knüpfen zu können. So beschreibt die 48-jährige Syrerin ihre Hauptkontakte in Österreich als eher oberflächlich und hauptsächlich aus der Grundversorgungseinrichtung oder den jeweiligen Kursen stammend. Die meisten ihrer Kontakte bestanden demnach zu anderen Syrer/inn/en. Auf die Frage, ob sie denn auch andere Araber/innen kenne, antwortete sie: „Nein, nein, wir treffen uns nicht miteinander. Naja, es gibt ein paar IrakerInnen. Warum treffen wir uns nicht mit anderen? Es ist hauptsächlich wegen der Sprache. In den Kursen gibt es ein paar Iraker/innen. Da sind auch Afghan/inn/en. [...] Da sind Leute aus unterschiedlichen Ländern. Wir gehen da hin und lernen einander kennen. Aber natürlich können wir nur mit den Iraker/inne/n sprechen. Vor allem mit den Afghan/inn/en können wir uns gar nicht treffen wegen der Sprache. Aber mit den Iraker/inne/n können wir uns manchmal treffen und besuchen einander." Die Sprachkurse selbst boten somit Möglichkeiten, um

12 Kursive Ausdrücke wurden im arabischen Interview auf Deutsch verwendet. 
Kontakte zu knüpfen und das eigene Netzwerk innerhalb der eigenen Sprachgruppe zu erweitern. Sie waren aber weder zwingend dem effektiven und schnellen Erlernen der deutschen Sprache dienlich, noch ermöglichten sie das Knüpfen sozialer Kontakte außerhalb der eigenen Sprachgruppe. Das obige Zitat macht deutlich, dass auch in Kursen, die nicht nach Sprachgruppen organisiert sind, Schwierigkeiten auftreten, Kontakte über die linguistischen Gruppen hinaus zu knüpfen, solange (noch) keine solide sprachliche Grundlage dafür existiert. Es besteht eine Ambivalenz zwischen dem Mangel an Möglichkeiten Personen anderer sprachlicher Hintergründe zu treffen und der Gruppenbildung entlang von Sprachkompetenzen in Situationen, in denen theoretisch auch übergreifende Kommunikation möglich sein könnte. Dies zeigt, wie komplex der Spracherwerb mit den Möglichkeiten zur Kommunikation und der Bildung interethnischer Freundschaften verknüpft ist.

\section{3 b Angebote für Geflüchtete}

Weitere Möglichkeiten der sozialen Partizipation bestanden in Angeboten für Geflüchtete im Rahmen von Integrationsmaßnahmen oder Flüchtlingsprojekten. Neben staatlich organisierten Kursen, wie etwa den ÖIF-Wertekursen, zählten dazu auch zivilgesellschaftliche Angebote, wie etwa Kochabende für Geflüchtete oder Sprachcafés, in welchen sich Geflüchtete sich mit Nichtgeflüchteten trafen, um sich auszutauschen und Konversationen (zumeist auf Deutsch) zu führen. Viele dieser Initiativen wurden von Ehrenamtlichen oder Hilfsorganisationen organisiert und gehörten zu den wenigen Situationen, in welchen Geflüchtete überhaupt Konversationen mit Österreicher/inne/n führen konnten.

Zudem gab es auch Initiativen Geflüchteter selbst, um soziale Netze aufzubauen und einander gegenseitig zu unterstützen. Ein junger Palästinenser berichtete, dass 2015 viele weitere Palästinenser/innen aus seiner Heimatsstadt Homs nach Österreich gekommen waren. Zusammen hatten sie einen Verein gegründet, in welchem sie nun auch andere Araber/innen aufnehmen wollten und ihn auf längere Sicht auch für Österreicher/innen interessant machen wollten. Auf die Frage nach der Aufgabe des Vereins erklärte er: „Wir sprechen miteinander. Was sind die Probleme? Was hast du gemacht? Was möchtest du? Wir tauschen unsere Erfahrungen aus. Leute, die schon Deutsch können, gutes Deutsch, gibt es kaum, vielleicht fünf oder sechs. Der Rest lernt noch. Die deutsche Sprache ist die oberste Priorität. Zuerst kommt die Sprache. [...] Wir unterstützen uns gegenseitig. [...] Die Hauptidee ist, dass wir uns gegenseitig helfen. Und wir versuchen einander immer zu helfen. Was für Hilfe? Zum Beispiel, wenn jemand ein Problem mit der Wohnung hat oder gesundheitliche Probleme. Zum Beispiel jemand, der die Sprache schon spricht, hilft jemandem, der sie noch nicht spricht. Systeme wie dieses oder einfach Zusammenkünfte, um Zeit miteinander zu verbringen. Vielleicht kennt jemand jemanden. Vielleicht kennt jemand Österreicher/innen und kann uns einander vorstellen.“ 
Für viele Geflüchtete war es wichtig, sich in Österreich einzubringen. Daher wurden nicht nur eigene Initiativen ins Leben gerufen, sondern auch die Übernahme von ehrenamtlichen Aufgaben angestrebt. Dafür waren verschiedene Gründe maßgeblich. Etwa wurde angeführt, dass sich die Übernahme von Verantwortung aus bestimmten Situationen ergab, wenn die Hilfe von Personen, die bereits besser Deutsch sprachen, benötigt wurde. Ein 25-jähriger Syrer beschrieb dies so: „Als ich nach Österreich kam, waren viele Leute neu hier. Wir waren alle in derselben Position. Wir waren neu in einem neuen Land. Aber jetzt bin ich schon seit drei Jahren hier. Wenn jemand Hilfe braucht, weiß ich was zu tun ist. Ich kann helfen. Bevor ich mit dem Studium angefangen habe, hatte ich viel Zeit und ich bin oft mit Leuten zum AMS gegangen, um dort zu übersetzen. Damals musste man sonst für einen Übersetzer zahlen." Ein anderer Syrer beschreibt, dass er sich freiwillig bei der Diakonie gemeldet hatte, um seine Zeit sinnvoll zu verbringen und nicht ins Nachdenken zu geraten. Wieder andere meinten, dass es sich um eine Strategie handelte, um Deutsch zu üben und Österreicher/innen außerhalb der Rolle als Geflüchtete zwanglos kennenzulernen. Ein 25-jähriger Syrer berichtete, dass er seinen ersten österreichischen Freund erst nach einem Jahr Aufenthalt gefunden hatte. Dieser Freund hatte ihn schließlich 2015 überredet, bei der Caritas als Ehrenamtlicher mitzuhelfen. Im Interview erzählte er: „Ich habe zuerst ehrenamtlich in einem Altenheim gearbeitet. Und dann 2015, mit der Flüchtlingskrise, habe ich mein Ehrenamt weiter ausgebaut. Dadurch habe ich viele neue Leute kennengelernt. Und dann, Schritt für Schritt, hat alles geklappt. [...] Ich habe das erste Jahr in Wien keine Deutschkurse gehabt. Ich habe von zu Hause aus mit Hilfe des Internets gelernt. Alleine gelernt. Deshalb habe ich gedacht, dass wenn ich mehr Kontakte haben will, ich ehrenamtlich aktiv werden muss. Deshalb habe ich mich eigentlich im Altersheim freiwillig gemeldet." Ein 34-jähriger Syrer beschrieb zudem, dass er an die österreichische Grenze fuhr und dort ehrenamtlich übersetzte. Er tat dies jedoch nicht primär, um Geflüchteten zu helfen, sondern um die Österreicher/innen zu unterstützen. Somit diente das Ehrenamt auch dazu, die eigene vorherige Position als Empfänger von Hilfsleistungen umzukehren und die persönliche Rolle in der neuen Umgebung umzudeuten bzw. neue Rollen einzunehmen.

\section{3c Online-Kommunikation}

Weitere wichtige Kommunikationsmöglichkeiten bieten soziale Medien. Für viele der Befragten stellen sie einen zentralen Aspekt ihrer täglichen sozialen Teilhabe dar.

Über Facebook, WhatsApp, Skype und Co. konnten viele die Kontakte nicht nur zu Familie und Freunden im Heimatland bewahren, sondern auch zu denen, die noch unterwegs waren, auf Familienzusammenführung warteten oder in einem anderen Land lebten. So erhielten soziale Medien Schlüsselfunktionen für das Familienleben und dienten dazu, bestehende soziale Kontakte wiederzubeleben (Khvorostianov et al. 2012, p. 590 f.). 
Der alltägliche Austausch über soziale Medien wurde wiederholt in fast allen Interviews beschrieben, wie beispielsweise von einer 33-jährigen Syrerin:

„Respondentin-R: Ich spreche jeden Tag mit meiner Familie und an fast allen Tagen mit meinen Freunden. Natürlich, da gibt es jeden Tag Kontakt, du weißt schon, über Facebook, WhatsApp und Internet generell. Auf Facebook bin ich auch mit meinen Freunden jeden Tag in Kontakt. [...] Ich versuche nicht über die Situation in Syrien nachzudenken. Aber das ist was Anderes. [...] Natürlich habe ich Familie in Syrien und ich mache mir Sorgen um sie. Ich habe ein Leben hier, aber auch ein Leben dort. Ich habe also meine Familie in Syrien, aber ich habe auch Geschwister außerhalb Syriens. Ich habe Geschwister in den Emiraten, in Saudi-Arabien und in Jordanien. Wir sind eine große Familie.

Interviewer-I: Seid ihr viel in Kontakt?

R: $\quad$ Wirklich viel. Wir haben eine WhatsApp-Gruppe, in welcher wir jeden Tag miteinander schreiben.

Dies führte jedoch auch dazu, dass aus der täglichen Verwendung sozialer Medien und der notwendigen Einbindung in bestehende soziale Netzwerke über Online-Medien ein Gefühl der Zerrissenheit zwischen der Präsenz in Österreich und der emotionalen und affektiven Einbindung andernorts entstand. Ein 30-jähriger Syrer beschrieb dies anschaulich: „Das Problem mit den Flüchtlingen ist, dass sie in zwei Welten leben. Da gibt es die Realität und die virtuelle Realität, Facebook und Twitter. Die Menschen leben hier in Österreich, aber die Familien, die Heimat und die Wurzeln sind Zuhause. Deshalb verbringen sie so viel Zeit auf Twitter und Facebook. Weil in ihren Heimatländern etwas passiert. Sie fühlen sich mehr mit ihrer Heimat verbunden als mit hier. Deswegen sind sie so viel auf Facebook. Das hat erst in den letzten sechs Jahren eingesetzt. Davor waren soziale Medien nicht so wichtig für uns, aber mit all den Kriegen ist nun unsere ganze Kommunikation und unsere Informationen auf Facebook. [...] Für mich ist es auch so. Meistens abends.“

Soziale Medien werden aber nicht nur benutzt, um mit Personen andernorts in Kontakt zu bleiben, sondern auch, um mit Personen in Österreich in Kontakt zu kommen. Beispielsweise existiert eine Facebook-Gruppe für mehr als 56.000 Arabisch-Sprecher/ innen in Österreich. Obwohl sie anfangs nur auf Syrer/innen ausgerichtet war, hat sie in den letzten Jahren großen Zulauf auch aus anderen arabischsprachigen Communities erhalten, die teilweise bereits seit mehreren Jahrzehnten in Österreich beheimatet sind. Diese ist allerdings nur eine von vielen arabischsprachigen aktiven Gruppen. Darin werden unter anderem Fragen gestellt, Rat gesucht oder Gegenstände verkauft. Es werden aber auch Auskünfte zu den aktuellen Geschehnissen in Syrien ausgetauscht oder Informationen aus und über Österreich diskutiert. Damit erfüllen viele dieser Konversationen nicht nur den Charakter der Informationsdissemination, sondern sind auch eine 
Art von Selbsthilfegruppe. Infolge des Wegfalls etablierter sozialer Strukturen, da diese entweder nicht mehr existent, außer Kraft oder nicht verfügbar sind, stellt das Internet für viele eine niedrigschwellige Alternative dar (MiKal \& Woodfield 2015, p. 1319). So können soziale Medien dazu beitragen, rasch ein unterstützendes Peernetzwerk zu etablieren. (vgl. MiKAL et al. 2013)

Aber nicht nur arabischsprachige Gruppen werden zur Kommunikation in Anspruch genommen, auch Gruppen für Helfer/innen und Freiwillige werden häufig genutzt. Die Kommunikation in diesen Gruppen findet hauptsächlich in deutscher Sprache statt. Anders als in Offline-Gesprächen konnten viele Interviewte in diesen Situationen auf Online-Übersetzungsprogramme zurückgreifen und somit Sprachbarrieren umgehen.

Soziale Medien ermöglichen darüber hinaus die Kommunikation mit Österreicher/ inne/n und wurden und werden daher häufig von Geflüchteten genutzt, um soziale Kontakte aufzubauen, wie dies ein 34-jähriger Syrer beschrieb: „Zuerst war ich in einer Gruppe, die Klosterneuburg hilft heißt. Danach war ich bei Tulln hilft. [...] Es gibt viele solcher Gruppen auf Facebook. Wenn du suchst, findest du viele solche Gruppen und viele neue Leute. Manche davon organisieren verschiedene Aktivitäten. Und durch diese Aktivitäten kann man auch wieder neue Leute kennenlernen." Allerdings handelte es sich häufig um Situationen, in welchen Hilfe gesucht oder Fragen gestellt wurden, welche dann von anderen Gruppenmitgliedern beantwortet wurden. Beispielsweise suchten Geflüchtete über Kontakte zu Helfer/inne/n nach Wohnungen, Kinderausstattung oder gebrauchten Möbeln oder holten Auskünfte zu Behörden und Anwälten ein. Somit basierte der betreffende Kontakt wiederum auf einem Machtgefälle zwischen demjenigen, der gibt, und demjenigen, dem gegeben wird. Die angesprochenen Konversationen beschränkten sich zumeist auf den virtuellen Raum und wurden nur selten in Offline-Kontakte übersetzt. Dies betraf sowohl Kontakte zu anderen arabischsprachigen Personen als auch jene zu Österreicher/inne/n.

Das Internet ermöglicht somit Kontakte, welche nur durch geringe geographische und linguistische Barrieren eingeschränkt sind, und erhöht die Kontrolle von Privatsphäre bei gleichzeitiger Reduktion sozialer Obligationen (MiKaL \& Woodfield 2015, p. 1322). Es können jedoch auch andere Barrieren umgangen werden, wie etwa Scham oder Sensibilität gegenüber bestimmten Themen (MALIK \& Coulson 2010, pp. 316 f.). WALTHER und BOYD beschreiben die Vorteile von Online-Kommunikation folgendermaßen: ,... candor (both less harsh and more forthright responses to problems), less negative judgement, reduced obligation to reciprocate support, less relational dependency, more immediate ability to seek support, greater expertise in the network, stigma management, intimacy, access, uninterrupted composition, more expressive communication, and anonymity.“ (WALTHER \& BOYD 2002, p. 179)

Über das Internet konnten sowohl bereits bestehende Netzwerke und Verbindungen aufrechterhalten als auch neue Kontakte geschlossen werden ohne notwendigerweise Machtunterschiede zu reproduzieren, auch wenn dies in manchen Foren dennoch 
geschah. Auf diese Weise wurde es zu einem wichtigen Fokuspunkt verschiedener Modi von Sozialität und kam den arabischen Konzepten wesentlich näher als bisher beschriebene Erfahrungen sozialer Partizipation in staatlichen Integrationsmaßnahmen oder zivilgesellschaftlichen Initiativen.

\section{Barrieren gegen soziale Kontakte}

Die am häufigsten genannte Barriere sozialer Teilhabe bestand in mangelnden Sprachkenntnissen, wie dies ein 27-jähriger Syrer beschreibt: „Wir sind alle Syrer, junge Männer. Wir sind alle Studenten und versuchen uns gegenseitig mit der Sprache zu helfen. Manche sind weiter als andere. Wir lernen zusammen und helfen einander. Wir können keine Freundschaften mit anderen schließen, deshalb versuchen wir miteinander zurechtzukommen und uns gegenseitig zu helfen. Wenn wir Fußball spielen, sind wir alle Syrer. Wir würden gerne andere Leute kennenlernen, aber wir haben keine Basis dafür."

An diesem Zitat wird deutlich, dass der Wunsch vorhanden war, soziale Beziehungen außerhalb der Sprachcommunity aufzubauen und z.B. mit Nicht-Syrer/inne/n Freizeitaktivitäten zu gestalten. Kontrastierend hierzu wurde auch verbalisiert, dass Personen der eigenen Sprachgruppe, obwohl präsent und ohne Sprachbarriere kontaktierbar, sogar gemieden wurden. Zudem war auch die erlebte Struktur des institutionalisierten Kontaktes mit der ansässigen Bevölkerung im Rahmen von Interaktionen mit Behörden oder Helfenden keine Art der Sozialität, die von den Befragten gewünscht wurde. Diese repräsentiert sogar eher ein Hindernis für soziale Kontakte. Im Folgenden soll auf die hintergründigen Logiken eingegangen werden, wieso sowohl der Kontakt zur eigenen Sprachgruppe als auch zu Ehrenamtlichen von vielen Befragten gemieden wurde. Und dies trotz der Notwendigkeit soziale Beziehungen aufzubauen. In beiden Fällen lässt sich die Art der entstehenden Sozialität als gegensätzlich zur gewünschten erkennen.

\section{$4 a \quad$ Kontaktvermeidung gegenüber der eigenen Sprachgruppe bzw. „ethnic community“}

Obwohl die eigene Sprachcommunity eine bedeutende Ressource sein kann, wird sie von vielen Personen bewusst gemieden. Dafür kann es unterschiedliche Gründe geben. Dieses Verhalten kann an schlechte Erfahrungen vor, während oder nach der Flucht geknüpft sein, wie etwa Konflikte verschiedener ethnischer oder religiöser Gruppen, aber auch an Korruptions- oder Gewalterfahrungen. Die Verfasser einer Vergleichsstudie in Deutschland fügen hinzu, dass Menschen keinen positiven Selbstwert mehr durch die Zugehörigkeit zu einer Gruppe erfahren und sie diese deshalb verlassen. (For- 
Schungsbereich beim SachVerständigenrat Deutscher Stiftungen für Intgration und MigRATiOn 2017, p. 76)

Häufig wurden intrinsische Gründe genannt, wie etwa, dass man nicht ständig an die Probleme von Geflüchteten in Österreich erinnert werden wollte. So berichtete etwa ein 53-Jähriger Syrer: „Ich habe nur ein paar syrische Freunde. Wirklich nicht viele. Sie zermürben dir nur den Kopf." Auf die Frage, ob ein 34-jähriger Syrer in Österreich syrische Freunde gefunden habe, antwortete er: „Ich habe nur zwei syrische Freunde. [...] Ich bin nicht gerne unter Syrer/inne/n. Ich glaube, wir hätten nur ein einziges Thema. Sie würden nur über die Situation hier sprechen und ich will darüber nicht reden. Ich habe selber genug damit zu tun. Ich hab' selber genug gesehen. Ich war Arzt in Dera'a $^{13}$ und ich habe die erste Person gesehen, die erschossen wurde. Alles hat in Dera'a angefangen. Ich war im Krankenhaus, als es angefangen hat. Von der ersten Sekunde an war ich da und habe Tausende gesehen, die ermordet wurden oder verletzt waren. Das ist genug für mich. Ich brauche niemanden, der darüber sprechen will." Die Meidung anderer Geflüchteter war somit eine häufige Strategie, um Gespräche über die aktuelle Situation in Österreich oder die Erlebnisse im Krieg und negative Erinnerungen auszublenden.

Oft zählen auch negative Erfahrungen mit anderen Gruppen oder Parteien in den Heimatländern zu den Gründen, weshalb Personen nicht gerne Kontakte mit anderen Geflüchteten pflegen möchten. Dies ist nach Bürgerkriegserfahrungen durchaus nicht unüblich. So beschrieb ein syrischer Kurde etwa, wie er sich in Syrien oft entscheiden hatte müssen, ob er sich als Syrer oder Kurde fühlte. „Früher hatte ich nicht viel Kontakt mit den Kurden. Meine Mutter ist Araberin und mein Vater ist Kurde. Ich bin also in der Mitte. Die Kurden sagen, ich sei Araber, die Araber sagen ich sei Kurde. Aber ich passe in beide [Kategorien]. In Syrien war das kein Problem, aber hier ist es manchmal eines. Zwischen uns gibt es eine Art Rassismus. Wenn die Syrer, die arabischen Syrer, wissen, dass ich Kurde bin, sehen sie mich anders. Aber wenn die Kurden wissen, dass meine Mutter Araberin ist, ändern sie auch ihre Meinung über mich. [...] Es ist ein häufiges Thema: Araber gegen Kurden. Wenn wir darüber reden, sind meine Ideen oft andere. Ich bin nicht ganz Araber und nicht ganz Kurde.“ (Syrer, 27 Jahre).

Auch die politische Zugehörigkeit und die Unterstützung unterschiedlicher Bürgerkriegsparteien konnten ausschlaggebend für den Ausbau oder die Meidung sozialer Kontakte mit bestimmten Personen der Community sein, wie etwa das folgende Zitat zeigt: „Wir haben so viele Probleme in Syrien. Viele Leute sind neutral, weder für noch gegen Assad. Und es gibt auch ehemalige Militärs des Assad-Regimes hier in Wien. Ich kenne viele Assad-Unterstützer. Wir haben Probleme, große Probleme mit den Politikern. Daher möchte ich keinen Kontakt oder Freunde unter den Syrern.“ (Syrer, 34 Jahre).

13 In Dera'a wurden 201115 Kinder festgenommen, welche beschuldigt worden waren, regimekritische Parolen an das Schulgebäude gemalt zu haben. Die Proteste aus Solidarität mit diesen Schülern werden als Beginn der Demonstrationen in Syrien angesehen. 
$\mathrm{Zu}$ den genannten intrinsischen Gründen treten jedoch auch extrinsische Gründe hinzu. Unter anderem waren sich die meisten Befragten der oft in den Medien formulierten „Angst vor Parallelgesellschaften“ bewusst und wollten dieser entgegenwirken. Der entstehende Integrationsdruck war ein weiterer Grund, wieso Befragte den Kontakt $\mathrm{zu}$ anderen Geflüchteten möglichst geringhalten wollten. Um zu zeigen, wie sehr man um die eigene Integration bemüht war, war es daher vielen wichtig, möglichst wenige Kontakte zu Landsleuten und dafür mehr Kontakte zu Österreicher/inne/n zu pflegen. Dabei lag bei vielen ein sehr essentialisiertes Verständnis davon vor, was es bedeutet „Österreicher/in“ zu sein, welches auch Angehörige einer zweiten oder dritten Generation von Neuösterreicher/inne/n ausschloss. Dies wurde auch gleichgesetzt mit der Möglichkeit des sozialen Aufstiegs. So fand etwa in einer jungen syrischen Familie eine Diskussion darüber statt, in welche Schule die sechsjährige Tochter gehen sollte. Während die Mutter die Ansicht vertrat, die Tochter sollte eine Schule in der Nachbarschaft besuchen, in welcher sie keine Außenseiterin sein würde, da es dort auch viele andere türkisch-muslimische Kinder gab, insistierte der Vater darauf, dass sie in eine weiter entfernte Schule gehen sollte. Dort wäre sie hauptsächlich unter österreichischen Kindern, würde die Sprache rascher lernen und hätte später bessere soziale Aufstiegschancen.

Die bewusste Kontaktvermeidung mit der eigenen (Sprach)community ${ }^{14}$ basiert auch darauf, dass viele Personen nicht als Geflüchtete kategorisiert werden wollen und darüber hinaus des Flüchtlingslabels überdrüssig waren. Vor allem wurde dieses Label auch als Hindernis für den eigenen Erfolg bzw. den Erfolg der Kinder in Österreich gesehen. Daher bevorzugen viele Befragte, dass die Kinder hauptsächlich mit Österreicher/inne/n verkehren sollten, wie das folgende Zitat belegt: „Zuerst, wenn du willst, dass deine Kinder gutes Deutsch lernen, sollten sie an einem Ort sein, wo jeder um sie herum Österreicher/in ist. In Wien bin ich mir nicht sicher, ob dies der Fall ist. In den Kindergärten und Schulen gibt es viele Kinder aus unterschiedlichen Ländern. Du kannst sie nicht alleine raus lassen oder im Garten spielen lassen. Du musst immer ein Auge auf sie haben“ (Syrer, 34 Jahre). Ähnlich antwortete ein 27-jähriger Syrer auf die Frage, ob es für ihn wichtig sei, Kontakte zu anderen Araber/inne/n zu haben: „Nein, nein. Ich hatte genug von denen. Ich wollte Deutsch sprechen. Das ist ja lustig, dass du das fragst." So wurde die Anwesenheit vieler Araber/innen eher als Hindernis für den eigenen Integrationsfortschritt in Österreich gesehen. Auf die Frage, ob es für einen 27-jährigen Iraker wichtig sei, dass es in seiner Nachbarschaft andere Araber/ innen gäbe, antwortete er: „Nein, nicht wirklich. Es wäre besser, wenn da keine wären. Ich möchte lieber Deutsch sprechen.“

Ein weiterer Grund für die Meidung anderer Geflüchteter liegt auch darin, dass Geflüchtete oft schlechte Erfahrungen im Zusammenhang mit der Hilfe seitens Personen der eigenen Community gemacht haben. Häufig wurde die entstandene Vulnerabilität von diesen ausgenutzt, um etwa auf dem Wohnungsmarkt Profit daraus zu schlagen. Dies führt dazu, dass Personen zu hohe Mieten zahlten, als Dritt- oder Viertuntermieter eine

\footnotetext{
14 Allerdings gibt es diesbezüglich auch Ausnahmen, wie etwa Gemeinden christlicher Minderheiten oder palästinensischer Syrer/innen.
} 
Matratze bezogen oder Privatpersonen Provision für die Vermittlung von Zimmern verlangten sowie auch Schwarzarbeit anboten. Ein 27-jähriger Iraker beschrieb etwa, warum er keine Arbeit bei den Arabern suchen möchte folgendermaßen: „Weil die Araber und die Türken uns nicht Vollzeit anstellen wollen! Zum Beispiel würde ich dann 15 oder 16 Stunden am Tag arbeiten und wäre nur für zwei Stunden angemeldet. Damit muss der Arbeitgeber weniger Steuern zahlen. Das ist gefährlich für uns. Und es ist [ein] wichtig[es] Thema. Es gibt so viele, die schon unregistriert arbeiten. Sie wollen das machen, damit sie endlich Geld verdienen." Der junge Mann wies mit Nachdruck auf die Ausbeutungsverhältnisse innerhalb der eigenen Community hin, in welcher es ein Bewusstsein der Abhängigkeit und Vulnerabilität der Arbeits- und Wohnungssuchenden gab.

Diese Entwicklungen produzierten Misstrauen in den Communities und führten oft zu einer komplexen und vielschichtigen Positionierung gegenüber Menschen derselben Herkunftsgruppe. Dies mag ein weiterer Grund dafür sein, warum die arabischsprachigen Communities in Österreich von den Interviewten eher mit Vorsicht betrachtet wurden. Neben schlechten Erfahrungen und der faktischen Ausbeutung durch Landsleute spielt auch der zunehmend kritische mediale Diskurs, der Geflüchtete und Migrierte vielfach als potentielle Kriminelle darstellt, eine erhebliche Rolle dabei, wie sich Communities entwickeln. Mehrmals wurde angemerkt, dass sich Geflüchtete vor kriminellen Geflüchteten fürchteten und bestrebt waren, dass ihnen diese möglichst fernblieben. Ein 34-jähriger syrischer Familienvater beschrieb dies so: „In Wien ist es nicht sicher. Ich fühle mich auch oft nicht okay, zum Beispiel, wenn ich mir nachts Zigaretten hole. Ich sehe dann viele Leute, die nicht okay sind und ich würde nicht wollen, dass meine Tochter oder meine Schwester ... Ich verstehe die Österreicher, wenn sie sagen: ,Wir finden nicht, dass unsere Töchter und Schwestern hier sicher sind." "Weiter führte er aus, dass er, um sich sicher zu fühlen, unterstützen würde, dass alle straffälligen Geflüchteten , auch wenn es sich nur um Bagatelldelikte handelte, sofort abgeschoben werden sollten. Misstrauen sowie aktuelle mediale und politische Debatten führen demzufolge auch zur Ablehnung und Diskriminierung der eigenen Community. Dies war nicht nur auf Seiten der neu angekommenen Geflüchteten der Fall, sondern auch eine gängige Erfahrung mit Personen, die bereits lange in Wien lebten, wie dies ein 53-jähriger Syrer beschrieb: „Einmal gab es eine Frau, die rassistisch war. Die Frau hatte zwei große Taschen. Sie waren sehr schwer. Ich habe meine Hilfe angeboten und es hat sich herausgestellt, dass sie Araberin war. [...] Sie hat mich nur komisch angesehen und gefragt: ,Was willst du? Was willst du von mir?' Ich war auf meinem Fahrrad. Ich sagte ihr, dass ich ihr nur helfen wollte. Ich sagte, ich könnte die Taschen auf dem Fahrrad transportieren. Sie sagte mir, ich solle weggehen und schrie mich an. Wäre es eine Europäerin gewesen, hätte sie mir bestimmt gedankt. Das passiert wirklich oft mit den Arabern hier.“

Das Resultat dieser Distanzierung und die Abwesenheit einer solidarischen Community jenseits der Online-Kommunikation in sozialen Medien zeigte sich in den folgenden Interviewpassagen. Ein 53-jähriger Syrer beschrieb, dass er keine Hilfe von anderen Syrer/inne/n oder Araber/inne/n in Österreich erhalten habe, da sich alle in derselben Situation befänden. Er sagte: „Zum Beispiel, du hast einen Job. Dann würde ich 
meinem Freund nicht davon erzählen, dass es eine Jobmöglichkeit gibt. Jeder sorgt sich nur um sich selber. Es ist anders als dort, von wo ich herkomme. Die Türken machen das hier schon. Sie kümmern sich umeinander und finden Jobs füreinander. Sie würden nicht zulassen, dass der andere arbeitslos bleibt. Aber die Araber machen das hier nicht." Eine 48-jährige Syrerin beschrieb darüber hinaus, wie die Isolation innerhalb der Community zur Vereinsamung führte. „Ich war daran gewöhnt, meine Familie um mich zu haben. Hier habe ich nur wenig Besuch. Bis jetzt geht es mir emotional nicht gut. Und Depression empfinde ich auch. [...] Ich fühle Depression und psychischen Druck." ${ }^{\text {"15 }}$ Auf die Nachfrage, ob sie jemanden hier hätte, der ihr helfen könnte, antwortete sie: „Hier habe ich niemanden. Hier haben wir kein Miteinander. Jeder hat mit der eigenen Situation zu tun. Alle Leute, die ich kenne, haben eine ähnliche Situation. Wir haben alle unsere Familien verlassen. Wir haben uns alle auf den Weg gemacht. Wir haben alle keine gute finanzielle Situation. Jedem geht es ähnlich. Ja, die Situation ist für alle gleich.“

In der aktuellen Migrationsforschung wurde nachgewiesen, dass Unterstützungsnetzwerke innerhalb der eigenen Herkunftsgruppe nicht nur problematisch gesehen werden dürfen, da ihnen ein gewisses soziales Segregationspotential innewohnt, sondern dass selbige durchaus eine wichtige Ressource gerade in der Anfangszeit darstellen, da sie Jobmöglichkeiten schaffen, aber auch Orientierungshilfen und emotionale Unterstützung bieten (vgl. Forschungsbereich BeIm SachVerständigenRat Deutscher Stiftungen für Intgration und Migration 2017, p. 76; DAmm 2009, p. 284). Infolge der Abwendung von der Herkunftsgruppe gehen auch wichtige Ressourcen gerade für die Anfangsphase des Aufenthaltes verloren, die hinsichtlich der Bewältigung anfänglicher Schwierigkeiten und sprachlicher Hindernisse zum Aufbau von Netzwerken auch über die Sprachcommunity hinaus sehr wichtig wären.

\section{$4 b \quad$ Österreicher/innen als Helfende}

Viele befragte Geflüchtete maßen dem Kontakt zu Österreicher/inne/n sehr hohe Bedeutung bei. ${ }^{16} \mathrm{Oft}$ wurden freiwillige HelferInnen als Bezugspersonen genannt. Obwohl betont wurde, dass die Arbeit der Freiwilligen sehr geschätzt wurde, gab es eine klare Reflexion darüber, warum diese Freiwilligen in einem Kontaktverhältnis zu den Befragten standen. In vielen Fällen war den Befragten bewusst, dass die Freiwilligen soziale Kontakte zu ihnen lediglich in Ihrer Position als Geflüchtete unterhielten und nicht aus persönlicher Sympathie oder Freundschaft. Hinsichtlich der Kontakte muss daher zwischen Unterstützung und persönlichen Freundschaften unterschieden werden (vgl. Forschungsbereich beim Sachverständigenrat Deutscher Stiftungen für IntgRATION UND MigRation 2017, p. 64). In genannter Studie wurde des Weiteren darauf

15 Die Respondentin benutzte die Termini ,ikti’ āb“ und „, dag̉t nafsī“ (bezüglich der Diskussion arabischer Taxonomien siehe den Beitrag von SCHIOcCHET in diesem Band).

16 Hierbei sind nicht nur Personen mit österreichischer Staatsbürgerschaft gemeint, sondern solche, welche sich in dieser Zeit in Österreich aufgehalten haben und von den Geflüchteten als Österreicher/innen wahrgenommen wurden. 
hingewiesen, dass Asylsuchende in Deutschland mit der ansässigen Bevölkerung zwar frühzeitig in einen Kontakt eintraten, jedoch ,handelt es sich meistens um Menschen, die sie ehrenamtlich unterstützen" (2017, p. 64). Dies führt eher selten zu einer persönlichen Begegnung auf gleicher Ebene, wie die Autor/inn/en anmerken, was bewirkte, dass Geflüchtete vor allem in der Anfangszeit eher sozial isoliert waren. Wenn Handlungsfähigkeit und Mündigkeit des Geflüchteten durch die Freiwilligen nicht ausreichend anerkannt werden, können sich paternalistische Handlungsmuster entwickeln (LINNERT \& BERG 2016, p. 19 f.).

Zudem verfestigt sich durch die Rollenzuschreibung in der Freiwilligenarbeit ein asymmetrisches Machtverhältnis zwischen den helfenden und unterstützten Personen. Dieses Machtgefälle war in den Initiativen wie Sprachcafés oder Kochabenden für Geflüchtete angesprochen worden, was zu einer Essentialisierung als „Geflüchtete“ führte und nicht selten einen weiteren Aspekt des Postmigrationsstresses darstellte. Bei den meisten Projekten und Organisationen, in welchen Geflüchtete aktiv waren und Kontakte zu Nichtgeflüchteten aufbauten, handelte es sich um Projekte, in welchen Sie in ihrer Rolle noch gestärkt wurden, anstatt einen gleichberechtigten Status aufbauen zu können. In vielen Fällen waren Helfende oder Lehrkräfte Ansprechpartner/innen für sehr spezialisierte rechtliche, aber auch finanzielle Fragen. In Studien zur ehrenamtlichen Arbeit wird dies häufig als eine Quelle für die Überforderung der Helfenden angeführt (LinNert \& Berg 2016, pp. 15, 19). Oft flossen die Grenzen zwischen Privatleben und Ehrenamt ineinander über (siehe ForschungsBereich BeIm SAChVERSTÄNDIGENRAT Deutscher Stiftungen für Intgration und Migration 2017, p. 77). So beschrieben viele Respondent/inn/en, wie freiwillige Sprachlehrende zu wichtigen Ansprechpartner/ inne/n wurden. „Ich habe diese Freundin. Sie ist 50. Sie und ihr Mann haben mir zwei Jahre lang Deutsch beigebracht. Ich war immer bei ihnen zu Hause, jeden Tag, privat. Das hat mir viel geholfen. Am Anfang war das wirklich schwer, weil sie keine richtige Lehrerin ist. Wir hatten Schwierigkeiten." Weiter erklärt der junge Mann, dass er sich aber in vielen Dingen an diese „Freundin“" wenden konnte und sie auch bei rechtlichen Fragen zu seiner Ansprechpartnerin wurde (Syrer, 25 Jahre).

Trotz dieser Hilfestellungen seitens der Zivilgesellschaft wurde von Geflüchteten das Bedürfnis nach sozialen Kontakten außerhalb institutioneller Vorgaben oder zivilgesellschaftlicher Angebote in der Geflüchtetenarbeit geäußert. Beispielsweise war bei vielen der Wunsch vorhanden, sich regelmäßig mit österreichischen Freunden und Familien zu treffen, und dass diese Kontakte nicht im Rahmen eines festen Programms stattfinden sollten. Etwa wünschten sich viele Geflüchtete, mehr Zeit mit den Nachbar/ inne/n verbringen zu können. Die gewünschten Beziehungen zu Österreicher/inne/n entsprachen demnach eher reziproken Verbindungen. Die Anonymität der Stadt und der Mangel an Nachbarschaftsgemeinschaft war für viele Personen unverständlich und repräsentiert ein beträchtliches Hindernis für soziale Integration. ${ }^{17}$ Auf die Frage, wie

17 In zahlreichen Studien zu Nachbarschaftsbeziehungen in Großstädten der Vereinigten Staaten von Amerika, aber auch Westeuropas zeigte sich, dass nur wenige Nachbar/inne/n in das engere soziale Netz befragter Personen fielen und dies mit der Größe der Stadt sogar noch abnimmt. Es 
denn die sozialen Kontakte in Österreich beschaffen seien, antwortete eine 48-jährige Syrerin: „Nein, wir haben keine Kontakte. Ich habe den Eindruck, dass die Leute hier nicht gerne viel miteinander zu tun haben. Wir waren in einem Gebäude, in welchem drei Wohnungen in jedem Stock waren. Wir haben dort eineinhalb Jahre gelebt und niemanden kennengelernt. Wenn wir jemanden gesehen haben, haben wir nur , Guten Morgen` gesagt. Nur das und dann sind sie gegangen. Du weißt, wir als Araber, wir kennen unsere Nachbarn. Und wir besuchen einander. Wir haben viele Nachbarn. Und natürlich haben wir auch unsere Verwandten in der Nachbarschaft. Meine Mutter, meine Schwester, die Schwester meiner Mutter. Und dann sind die Nachbarn und die Familie eh oft dieselben. Wir haben die Pflicht uns gegenseitig umeinander zu kümmern. [...] Wir trinken Kaffee miteinander. Oder wir laden uns gegenseitig zum Frühstück ein. [...] Aber hier scheinen sie nichts miteinander zu machen. Wie soll man sich denn hier integrieren?" Im Anschluss daran beschrieb sie, wie ihr Mann in Syrien mit anderen Nachbarn auf dem Korridor oder vor dem Haus Zigaretten geraucht hatte und zusammensaß. Wenn er nun in Österreich vor die Tür ging um zu rauchen und dabei auch Kontakte zu knüpfen, lernte er jedoch keine neuen Nachbar/inne/n kennen. Die Frau erklärte sich dies folgendermaßen: „Ich glaube, die Leute hier sehen einander nicht gerne. Sie essen auch nicht gerne miteinander. Man sagt uns, wir sollen uns integrieren. Wir Araber, wir lieben Integration. [...] Du weißt, wir Araber lieben es, diese Dinge miteinander zu tun und miteinander zu reden und einfach sozial zu sein. Aber hier mag man diese Integration nicht so." Für viele Befragte war der Kontakt zu den Nachbar/ inne/nsahen im Kontakt zu den Nachbar/inne/n die erste und unmittelbare Instanz sozialer Teilhabe. Anders als in Österreich gibt es allerdings in vielen Teilen der arabischen Welt nur eine geringe residentielle Mobilität. ${ }^{18}$ Wohnungen werden zum Teil nach der Eheschließung bezogen und in den meisten Fällen nicht mehr verlassen. Dadurch entstehen in der Nachbarschaft lebenslange Beziehungen. Durch schwächer entwickelte wohlfahrtsstaatliche Leistungen entfallen viele dieser Aufgaben nicht nur auf Angehörige, sondern vor allem auch auf die Nachbar/inne/n (vgl. KAMAL 2019). Innerhalb der Nachbarschaft werden finanzielle Ressourcen für Begräbnisse oder Hochzeiten mobilisiert sowie gegenseitige Unterstützung bei der Kinderbetreuung, beim Ausbau ökonomischer Netzwerke oder andere Hilfeleistungen lukriert. Daher wird in empirischen Studien über Nachbarschaften im arabischen Kontext oft kein Unterschied zwischen Familien- und Nachbarschaftsbeziehungen gemacht, da beide Kategorien in die soziale Sphäre der qaraba fallen (vgl. EICKELMAN 2002). Intensive nachbarschaftliche Beziewäre denkbar, dass diese Erkenntnisse auch auf Wien übertragbar sind (vgl. FISCHER 1982; THоMÉSE \& VAn Tilburg 2000; VAn der Poel 1993).

18 Hierin besteht ein wesentlicher Unterschied zu anderen Gruppen, die bereits vor der Ankunft in Österreich Migrationserfahrung aufwiesen. Viele türkische Familien aus ländlichen Gegenden haben mehrmalige Übersiedlungen von ruralen in urbane Siedlungstypen hinter sich, bevor sie nach Österreich kommen. In Wien wiederum lag die Eigentumsquote 2017 bei 19,1 \% (STATISTIK AUSTRIA 2018), während in den meisten arabischen Ländern eine dauerhafte Residenz bei der Eheschließung bezogen wird und die Eigentumsquote im Vergleich zu Wien äußerst hoch ist. Vor den Auswirkungen des Krieges in Syrien und den damit verbundenen internen Vertreibungen gab es somit kaum residentielle Mobilität. 
hungen und regelmäßige soziale Interaktionen werden daher nicht nur als pragmatische Notwendigkeit, sondern vor allem auch als eine ethische Verpflichtung angesehen. Die als mangelhaft wahrgenommenennden Möglichkeiten nachbarschaftlicher Beziehungen in Wien wurden von vielen Befragten als eine beträchtliche Barriere für das Eingehen sozialer Kontakte beschrieben. So erzählte eine alleinerziehende Syrerin, dass es in Syrien üblich sei, oft und viele Besuche abzustatten. „Aber in Österreich sind die Leute normalerweise sehr beschäftigt mit ihrer eigenen Situation. Und das betrifft nicht nur die Österreicher/innen, sondern auch die Syrer/innen. Jeder hat die ganze Zeit Termine, Kurse und so. In Syrien hat man mehr Zeit. Die Nachbarn essen miteinander oder laden sich zum Kaffee ein. Das ist wichtig. Man unterstützt sich gegenseitig. Hier kommt viel Unterstützung von der Regierung. In Syrien ist das nicht so viel.“ (Syrerin, 33).

Keine engen nachbarschaftlichen Kontakte einzugehen bedeutet daher im arabischen Kontext nicht nur über ein wichtiges soziales Netz nicht zu verfügen, sondern zugleich auch eine gesellschaftliche Marginalisierung, die oft zusätzlich sozial negativ sanktioniert wird (vgl. KAMAL 2019). Die Anonymität der Nachbar/inne/n in Wien und die mangelnden Informationen über existierende Möglichkeiten, mit der Umgebung in Kontakt zu treten, stellen nicht nur ein erhebliches Hindernis für die soziale Integration dar, sondern bedingen zugleich auch ein beträchtliches Frustrationspotential. ${ }^{19}$ Vor allem ältere Personen litten darunter, dass niemand Zeit hatte. Auch die eigenen Angehörigen waren durch staatliche Integrationsverpflichtungen zu beschäftigt, um sich um sie zu kümmern.

Ein erfülltes soziales Leben gab es für viele Geflüchtete in Österreich kaum. Ein 72-jähriger Syrer beschrieb demnach das „Wesen“ der österreichischen Gesellschaft als „zurückgezogen“: „Der Status der Gesellschaft hier ist schwach. Wären wir beide von hier, würden wir uns nicht besuchen. Ich würde nicht zu dir nach Hause kommen und du nicht zu mir. Ich würde dir nichts zu essen anbieten. Ich würde dir nicht ,Guten Morgen“ sagen. [...] Die Österreicher/innen besuchen einander nicht und grüßen sich nicht. Morgens, wenn ich meine Türe öffne und mein Nachbar auch, dann sehen wir uns von Auge zu Auge aber wir grüßen einander nicht. Nie! Das finde ich hier wirklich komisch. Es wäre doch normal, sich einen guten Morgen zu wünschen [...] Die Österreicher/innen sind sehr nett. [...] Aber ihnen fehlt es an sozialem Leben. Ihnen fehlt es an sozialem Leben und gut verstrickten Familienbanden. [...]"“

Ein 53-jähriger Syrer beschrieb, wie er stets versucht, sich zu integrieren und auch Kontakte mit Österreicher/innen einzugehen, was ihm jedoch nicht gelingt. Er berichtet: „Man muss sich in die Gesellschaft integrieren. Aber wie soll das gehen? Jeder ist immer mit sich selber beschäftigt. Da gibt es nichts, in das man sich integrieren könnte. Zum Beispiel, dass dein Nachbar bei dir klopft und dich zum Kaffee einlädt, das würde hier einfach nicht passieren. [...] [In Damaskus] helfen alle Nachbarn einander. Jeder kommt, jeder hilft. Aber hier gibt es das nicht. Euer Brauch hier ist, dass jeder für sich

19 Hier lässt sich die Überlegung anschließen, ob der mangelnde Ausbau nachbarschaftlicher Beziehungen in Großstädten nicht generell ein Problem des großstädtischen Lebens darstellt, welches durch Migration und Fluchtdynamiken augenscheinlicher geworden ist. 
ist. Ihr mischt euch nicht untereinander. ${ }^{20}$ Wie könnten wir das dann tun? Wenn du deinen Nachbarn siehst, würdest du ihm nicht mal ,Guten Morgen' wünschen. Niemand grüßt hier irgendjemanden.“

\section{4c Machtgefälle und Essentialisierung}

Kommunikation und soziale Beziehungen zu Österreicher/inne/n oder offiziellen Vertreter/inne/n österreichischer Institutionen wurden vorwiegend auf einer formalen Ebene erlebt, d.h. im Kontext von Ämtern, wie etwa dem AMS, dem Bundesamt für Fremdenwesen und Asyl (BFA), oder Leiter/inne/n von Unterkünften und NGOs oder als Instrukteure bzw. Lehrkräfte. Diese Art von Sozialität basiert auf einer ungleichgewichtigen Machtposition, in welcher Geflüchtete Österreicher/innen hauptsächlich als wertend, entscheidend oder instruierend erlebten. So antwortete ein 30-jähriger kurdischer Syrer auf die Frage, ob er immer mit Respekt behandelt wurde: „Nein, weißt Du. Das ist ein sehr wichtiger Punkt, den man in der Flüchtlingsthematik mitdenken muss. Leute, die im Flüchtlingssektor arbeiten, arbeiten mit Mitleid und nicht mit Respekt. Weißt Du, was ich meine? Wir werden bemitleidet. Es ist Mitleid. Aber das sollte es nicht sein. Es ist wie mit arm und reich. Respekt heißt, dass man einander auf Augenhöhe begegnet. Abgesehen davon sind die Österreicher/innen nett."

Aber gerade die Möglichkeiten einander auf Augenhöhe zu begegnen wurden strukturell erschwert. Zum einen waren reale Möglichkeiten sozialer Partizipation durch sprachliche und finanzielle Limitationen eingeschränkt, darüber hinaus aber auch durch Vorurteile und den eigenen Rückzug. Ein 27-jähriger Iraker beklagte zum Beispiel, dass er keine Kontaktgelegenheiten zu Personen in seinem Alter habe, da er weder Schwimmbäder und Konzerte noch Diskotheken besuchen könnte. Seine 23-jährige syrische Freundin fügte hinzu, dass dies aber nicht nur ein Problem von jungen Männern sei, sondern dass auch ihre syrische Freundin keinen Einlass in Diskotheken erhielt. Der junge Mann führte dies darauf zurück, dass die Freundin wohl ein Kopftuch trage. Aber seine Freundin erwiderte: „Nein, nein, sie hat kein Kopftuch. Der Türsteher hat nach ihrem Ausweis gefragt. Als er gesehen hatte, dass der grau war, hat er sie nicht rein gelassen. [...] Sie ist ein Flüchtling. Das hat dem Türsteher gereicht.“ Auf die Frage, wo man denn dann Gelegenheit hätte gleichaltrige Österreicher/innen kennenzulernen, meinte der Iraker, dass man keine andere Möglichkeit habe, als zu Hause zu bleiben, da man auch nicht ins Schwimmbad gehen könnte und die Fitnesscenter der Österreicher/ innen zu teuer seien.

Zudem erklärten viele Respondent/inn/en der sozialen Kategorie als Geflüchtete überdrüssig zu sein. Soziale Partizipation und Kommunikation fanden also innerhalb eines Machtgefälles statt. Dieses führt auf Dauer zu einem Gefühl der Entfremdung und einer veränderten Selbstwahrnehmung als Person, nämlich als defizitär, als ein Mensch, dem noch etwas fehlt. Dieses Gefühl vermischte sich mit der ständigen Erwartung von 20 Die befragte Person benutzte das arabische Wort Mazaja. 
Dankbarkeit seitens der Aufnahmegesellschaft. Diese als erzwungen empfundene Dankbarkeit und damit paternalisierende Abhängigkeit war für viele Befragte unerträglich und nicht mit dem eigenen Sinn für persönliche Integrität zu vereinbaren. Eine 33-jährige Syrerin erklärte, dass sie hauptsächlich syrische Freunde hätte, die sie allesamt erst in Österreich kennengelernt habe. Österreicher/innen kenne sie hauptsächlich über Hilfsinitiativen. „Die meisten meiner Freunde kenne ich über die Kurse: Integrationskurse, Deutschkurse. Und die Österreicher, ich kenne sie über meinen Bruder oder über das Camp, wenn sie gekommen sind und uns dort geholfen haben." Im Umgang mit den Personen in den Flüchtlingsinitiativen erlebten viele Befragten auch eine gewisse soziale Distanz, wenn Geflüchtete den Kontakt auf eine persönliche Ebene bringen wollten. So blieben etwa persönliche Telefonate oder Besuche aus und der Kontakt beschränkte sich lediglich auf den Rahmen der jeweiligen Initiative. So berichteten zwei Schwestern aus Syrien, 18 und 19 Jahre alt, wie sie regelmäßig ihre Sonntage in einem Sprachcafé verbrachten, welches im Anschluss an einen katholischen Gottesdienst in einer katholischen Wiener Pfarre stattfand, dass sie abgesehen von den Teilnehmer/inne/n im Sprachcafé kaum Kontakte zu Österreicher/inne/n hatten. Auch wurde deutlich, dass zwischen der demographischen Zusammensetzung der Ehrenamtlichen und jener der Besucher/inne/n beträchtliche große Unterschiede bestanden:

\author{
„Interviewer- I: Ist das Sprachcafé wichtig für euch? \\ Ältere Schwester: Ja, es hilft mir sehr beim Deutsch sprechen. [...] \\ Jüngere Schwester: Alle Leute dort sind unsere Freunde. Es gibt dort drei oder \\ vier österreichische Familien. \\ I: $\quad$ Sind sie so alt wie ihr? \\ Jüngere Schwester: Nein, sie sind alle älter. Aber manche haben Kinder, die um \\ die 30 sind. Manchmal kommen sie mit.
}

Viele der ehrenamtlichen Initiativen wurden demnach von älteren Personen getragen. Dennoch wurden sie meist von jüngeren Geflüchteten wahrgenommen. Zudem war der Austausch in den Sprachcafés oft mit recht unterschiedlichen Erwartungen verbunden. Während es sich für österreichische Teilnehmer/innen um eine von Art Wohltätigkeit oder ein Ehrenamt handeln konnte, beschrieben die beiden Schwestern die anwesenden österreichischen Familien als ihre „Freunde.“ Ein ähnliches Missverständnis bezüglich der gegenseitigen sozialen Beziehung stellt die Erfahrung eines 35-jährigen syrischen Palästinensers dar. Er beschreibt: „Da gibt es diesen alten Mann im Sprachcafé. Sein Name ist Hans. Ich habe ihn ein paar Mal getroffen und dann nach seiner Nummer gefragt. Ich dachte, wir könnten uns vielleicht auch so mal treffen. Aber er war sehr verwundert und meinte: ,Wieso sollte ich dir meine private Nummer geben? Wir sehen uns doch eh hier regelmäßig. “ ' Daran wird deutlich, dass das Ehrenamt in der geschilderten Situation als soziales Engagement angesehen wurde und nicht als eine darüberhinausgehende Investition in eine private Freundschaft. Für den 35-jährigen Palästinenser war diese Ablehnung der persönlichen Freundschaft allerdings sehr beschämend, wie er berichtete, und der maßgebliche Grund, das Sprachcafé nicht mehr 
zu besuchen. Missverständnisse dieser Art repräsentieren keine Seltenheit im Kontext der ehrenamtlichen Arbeit.

Für viele Personen resultierte aus der beträchtlichen medialen Aufmerksamkeit und dem Sonderstatus ein gewisses Gefühl der Übersättigung. Dies beschrieb ein 27-jähriger Syrer folgendermaßen: „Leute sehen mich nur als Flüchtling, als Fremden. Sie sehen mich voller Mitleid an, weil ich mein Land kriegsbedingt verlassen habe. Es gibt viele Leute, die mitfühlen. Andere sehen, dass ich aus Syrien komme, aus der Armut. Und sie denken, ich komme um ihr Geld zu nehmen. Klar gibt es auch solche.“

Dies führte für viele auch zum sozialen Rückzug, wie dies etwa ein 34-jähriger Syrer beschreibt: „Ich habe mich entschieden alleine zu sein, weil ich Kopfweh von den Leuten bekomme, wegen dem, was sie sagen und was sie so diskutieren. Auch jetzt, wenn ich hier mit dir sitze, sitze ich hier als Flüchtling. Du wolltest mich als Flüchtling interviewen. Wenn ich zu [einer NGO] gehe, tue ich das, weil ich als Flüchtling Hilfe brauche. Wenn ich ins Internet gehe, wenn ich etwas anhöre oder die Nachrichten lese, dann ist alles immer über dich. Jeder spricht über dich." Die Essentialisierung der Geflüchtetenrolle und zugleich das In-den-Hintergrund-Treten der Persönlichkeit im Rahmen der öffentlichen Wahrnehmung war ein Knackpunkt, der für viele Personen zu Frustration und Rückzug geführt hat. Das Wissen, dass viele soziale Kontakte zu Österreicher/inne/n gerade auf dieser Rolle aufbauten, wurde häufig thematisiert und war für viele Personen der Grund, weshalb sie keine tiefergehenden und tragfähigen Beziehungen aufbauen konnten.

Gerade die Wahrnehmung als „Flüchtling“ ohne persönliche oder biographische Hintergründe war für viele Personen eine sehr schwierige Situation. Vor allem Befragte, die vorher einen gewissen gesellschaftlichen Status innehatten und diesen infolge ihrer Flucht verloren hatten, konnten schwer mit dieser neuen Rolle umgehen. Ein 34-jähriger ehemaliger Arzt sagte: „Es ist so schwer ein Flüchtling zu werden. Wenn du dein Zuhause verlässt, lässt du ein Stück deiner Würde zurück. Weil du danach nichts mehr bist. Du hattest einen Job, ein Haus, alles, in deiner Heimat. Und dann kommst du hierher und hast nichts. Du sprichst nicht mal die Sprache. Du beginnst dich so zu fühlen, als hättest du keine Würde mehr. Leute bekommen ihre Würde zurück, wenn sie arbeiten. Solange das Geld von der Regierung kommt, haben sie keine Würde." Auf die Frage, was es denn für Konsequenzen habe, wenn Leute sich würdelos fühlten, antwortete er: „Das ist, was gerade passiert. Sie können sich nicht integrieren. Das ist das Problem. Wenn du in einer Gesellschaft lebst, ohne respektiert zu werden, ohne Würde, ohne Sprache, ohne Freunde, dann fühlst du, dass du hier nicht hergehörst. Dann ist es unmöglich sich zu integrieren."

Ein weiteres soziales Qualitätsmerkmal, welches im Umgang mit Österreicher/ inne/n häufig vermisst wurde, war Wertschätzung. So erklärte ein 27-jähriger Syrer, wie wichtig diese für sein soziales Leben in Österreich sei: „Wertschätzung ist das Allerwichtigste. Ich bekomme das von meinen Freunden, aber nicht von der Gesellschaft selbst. Ich meine, Wertschätzung erhält man durch die kleinen Dinge im täglichen 
Leben, nicht durch große Dinge. Ich habe Kern getroffen. Er gab eine Vorlesung in der Kreisky-Villa. Ich meine, ich will dir ein Beispiel geben. Es kommt durch die kleinen Dinge. Wertschätzung! Kern hat mir gesagt, dass mein Deutsch schon so gut ist. Er war sehr zufrieden mit mir. Und am selben Tag bin ich in die Trafik gegangen, um Tabak zu kaufen. Mein 5-Euro-Schein war noch sehr neu. Ich habe gezahlt und die Person hat mich gefragt: ,Was macht ihr mit eurem Geld?' Ich wollte sie fragen, wen sie mit ,ihr meint und sie sagte: ,Na, ihr!' Ich wollte nicht diskutieren und bin gegangen. Wertschätzung oder nicht kommt durch diese kleinen Situationen und nicht durch ein Treffen mit dem Bundeskanzler. [...] Ich treffe ihn nicht jeden Tag, aber ich treffe diese anderen Personen jeden Tag.“

\section{Conclusio}

Für viele Befragte bestand eine beträchtliche Herausforderung in der mangelnden Verfügbarkeit von Ressourcen, um mit dem Postmigrationsstress und dem Integrationsdruck in Österreich zurechtzukommen. Gerade der Mangel an erfüllender Sozialität in den Sozialkontakten zur ansässigen Bevölkerung in Kombination mit dem Zurechtkommen mit der neuen Rolle als Geflüchtete stellte für viele eine erhebliche Belastung dar. Mangelnde Kommunikation, Diskriminierungserfahrungen, aber auch die Sorge um die Familie in den Kriegs- oder Transitländern, finanzielle Schwierigkeiten und Barrieren bezüglich des Aufbaus von Kontakten oder der Annahme von Hilfe waren besonders häufig genannte Postmigrationsstressoren. Vergleichbare Studien mit Geflüchteten verweisen auf die Rolle der Ungewissheit, das nicht ausreichende Verständnis der Sprache des Aufnahmelandes sowie Schwierigkeiten sich in bestehende Systeme einzufügen (Mikal \& Woodfield 2015, p. 1321). Dieselben Autor/inn/en betonen, dass dies zu Depressionen, Angst, Isolation sowie zu psychosomatischen Erkrankungen führen kann (2015, p. 1321).

Vielen Respondent/inn/en war es ein wichtiges Anliegen, aus der Hilfsbedürftigkeit und der Position als „Empfänger“ von Hilfestellungen auszusteigen und eine selbstbestimmtere Art der Sozialität zu erreichen. Sie gaben an, gerne anderen Personen ihre Hilfe anbieten zu wollen, Nachbar/inne/n und befreundete Österreicher/innen nach Hause einzuladen oder anderen eigene Fertigkeiten zu vermitteln. Die Verfasser der Vergleichsstudie in Deutschland gelangten zu einem ähnlichen Ergebnis und geben an, dass „diese Wechselseitigkeit den Menschen erlaubt, sich selbst als handlungsfähig wahrzunehmen, und ihnen das Gefühl, echter' Teilhabe vermittelt, im Sinne eines sozialen Miteinander und Füreinander." (Forschungsbereich beim SAchVerständigenRat DeutSCHER Stiftungen für Intgration und Migration 2017, p. 74). Viele der bisher erlebten Kontakte zu Behörden oder Fachpersonal in Institutionen sowie Hilfeleistungen von Freiwilligen glichen nicht der erwünschten Sozialität persönlicher Interaktionen auf gleicher Augenhöhe. 
Sozialität als komplexer Prozess, durch welchen Personen mit der sie umgebenden sozialen Welt in eine Beziehung treten oder sich ablösen (vgl. Long \& Moore 2013, p. 12) scheint ein adäquateres Konzept als das der sozialen Integration zu sein, um die Hintergründe für das Zu- oder Abwenden hinsichtlich sozialer Kontakte sowie die damit einhergehenden Motivationen und Wünsche zu verstehen. Innerhalb einer dynamischen Matrix sozialer Beziehungen werden unterschiedliche ethische und normative Wertvorstellungen bezüglich der Art von Beziehungen und der Kategorien von Interaktionspartner/inne/n, mit welchen man in Beziehung tritt, entworfen (vgl. Long \& Moore 2013, p. 13). Vor allem durch die Angst vor der Bildung von „Parallelgesellschaften“ in Österreich und den vermehrten Integrationsdruck seitens der neuen Regierung ergab sich für viele Befragte eine unterschiedliche Wertigkeit gegenüber Beziehungen zu Österreicher/ inne/n, Personen der eigenen Sprachgruppe oder anderen Zugewanderten. Fragen nach diesen Wertigkeiten dürfen nicht losgelöst von aktuellen politischen Diskursen gesehen werden. Der Integrationsdruck, aber auch die derzeitigen Flüchtlings- und Integrationsdebatten in Österreich und den Nachbarländern beeinflussen, welche Wertzuschreibungen und Vorstellungen sozialer Erwünschtheit von Kontakten Geflüchtete wahrnehmen und auf welcher Basis sie selbst soziale Kontakte forcieren oder meiden.

So ergab sich aus den Interviews, dass besonders bestimmte Arten von Sozialität, nämlich jene, welche auf Gegenseitigkeit und auf Partizipation ausgerichtet sind, herbeigesehnt wurden. Für viele Respondent/inn/en entsprach dies der erwünschten künftigen Art des sozialen Austausches vor allem mit den Nachbar/inne/n, sobald das notwendige Niveau an Deutschkenntnissen vorhanden sei. Für andere hingegen, welche bereits gut genug Deutsch sprachen und die diese soziale Reziprozität bisher dennoch nicht finden konnten, repräsentierte dies eine utopische Alternative mit hohem Frustrationspotential.

Es zeigte sich ein Spannungsverhältnis zwischen den Arten der Sozialität, welche aus den vorgeschriebenen Integrationsmaßnahmen und der gewünschten Art der reziproken sozialen Teilhabe resultierten. Dieses Spannungsverhältnis lässt sich in vielen der beschriebenen Möglichkeiten sozialer Partizipation wiedererkennen und bietet eine Interpretationshilfe, um die strukturellen und sozialen Barrieren gegen soziale Kontakte zu verstehen. Die aus der derzeitigen Integrationsdebatte und dem aktuellen Flüchtlingsservicesektor entstehende Sozialität ergibt vor allem eine Top-down-Kommunikation, welche auf einem Machtgefälle basiert und bestimmte strukturelle Prozesse bedingt, welche den (Wieder)aufbau tragfähiger sozialer Netze eher erschwert als fördert. Diese Prozesse ließen in den Interviews Tendenzen erkennen, welche Isolation und Abgrenzung vorantreiben, zusätzlichen Postmigrationsstress auslösen und ein hohes Frustrationspotential aufbauen.

Hervorzuheben ist jedoch auch die Fragilität dieser gewünschten Sozialität, welche gleichzeitig von Anerkennung, aber auch von Makrostrukturen politischer Ökonomie abhängt und ein hohes Frustrationspotential beinhaltet (vgl. Allison 2013). Befragte wünschten sich mehr als lediglich eine formale Integration durch das Erfüllen 
der Integrationsauflagen. Ein 27-jähriger Syrer machte klar: „Weißt du, es wird nicht immer so bleiben. An einem gewissen Punkt werden die Leute aufhören es immer wieder zu versuchen [Teil der Gesellschaft zu werden]. Sie werden keine Energie mehr haben, wenn von der anderen Seite nichts kommt. Und vor allem, die neue Regierung, die Art der Integration, die sie wollen, ist keine soziale. Sie wollen nur [das]: es ist nur Arbeitsintegration. Sie wollen nur, dass man schnell arbeitet. Aber daran werden die Leute zerbrechen." Nun sind vor allem die Vereine und Nachbarschaftsinitiativen gefragt, um diesen Wunsch nach gleichberechtigter Sozialität aufzufangen. Dabei sollte es sich jedoch nicht um Angebote handeln, die Geflüchtete in ihrer Rolle als Geflüchtete in den Verein inkorporieren, sondern als gleichberechtigte Teilnehmer/innen und unabhängig von ihrer Herkunft.

Eine weitere Erkenntnis aus den Interviews liegt darin, dass das Bedürfnis nach reziproken Unterstützungen auf nachbarschaftlicher Basis, welches nachdrücklich betont wurde, auch zu einer grundsätzlichen Verbesserung der Nachbarschaftbeziehungen in österreichischen Großstädten beitragen könnte. Seit längerer Zeit steht die Verbesserung nachbarschaftsbasierter sozialer Beziehungen im Zentrum des Interesses von Stadtplaner/inne/n in westeuropäischen Kontexten (vgl. Forrest \& KEANS 2001). So weisen Van Den Berg und Timmermans darauf hin, dass gute Nachbarschaft nicht nur die Lebensqualität in der Stadt verbessern, sondern auch unterstützend wirken kann, um mit einer Reihe an urbanen Herausforderungen zurechtzukommen, wie etwa der Nutzung des öffentlichen Raumes, Armut oder Arbeitslosigkeit zu bewältigen (2015, p. 56). $\mathrm{Zu}$ diesem Zweck werden neben „harten“ Maßnahmen, wie der Restrukturierung des Wohnungsmarktes, auch ,weiche“ Maßnahmen, wie die Stimulierung sozialer Initiativen innerhalb urbaner Viertel gefördert. Diese Maßnahmen dienen der Mobilisierung aktiver Bürger/inne/n, die Verantwortung in Initiativen und gegenüber ihrer räumlichen Wohnumgebung zu übernehmen (HoEkstra \& DAHLVIK 2017, p. 442, siehe auch NeWMAN \& TONKENS 2011). Um Nachbarschaftsförderung auf zivilgesellschaftlicher Ebene diesen Vorstellungen entsprechend zu ermöglichen, ist ein Umdenken nötig, welches das positive Gestaltungspotential Geflüchteter fördert, anstatt mediale und politische Rückgriffe auf veraltete Denkkonstrukte, wie etwa jenes der „Parallelgesellschaft“ vorzunehmen. Aus den qualitativen Interviews mit Geflüchteten wurde ersichtlich, welche Qualität der Sozialität sie sich im nachbarschaftlichen Umgang wünschen. Gerade die Potentiale dieser Unterstützungsnetzwerke, welche aus lokaler Nähe, pragmatischen Überlegungen und ethischen Verpflichtungen entstehen, um Versäumnisse staatlicher Fürsorgepflichten in arabischen Kontexten auszugleichen, bilden eine wichtige Ressource, die auch in Wien zur Verbesserung des innerstädtischen sozialen Klimas beitragen könnte. Auf dieser Einsicht beruhend liegt es nahe, ein Umdenken von problem- zu potentialorientierten Diskursen zu fordern.

So lässt sich dies mit einem Zitat eines jungen Syrers zusammenfassen, wenn er erklärt: „Ich möchte als Mensch wahrgenommen werden und nicht für immer ,der Geflüchtete" bleiben.". 


\section{Bibliographie}

Ager A. \& Strang A. (2008), Understanding Integration: A Conceptual Framework. In: Journal of Refugee Studies 21 (2), pp. 166-191.

Allison A. (2013), A Sociality of, and Beyond, 'My-home' in Post-corporate Japan. In: Long N.G. \& Moore H.L. (eds.): Sociality. New directions. New York, Berghahn (Wyse Series in Social Anthropology, 1), pp. 117-132.

Aміт V. (ed.) (2015), Thinking through Sociality: An Anthropological Interrogration of Key Concepts. New York, Oxford, Berghahn.

Aмiт V. et al. (2015), Introduction. Thinking through Sociality: The Importance of Midlevel Concepts. In: Амiт V. (ed.): Thinking through Sociality: An Anthropological Interrogation of Key Concepts. New York, Oxford, Berghahn, pp. 1-19.

Aus Politik und Zeitgeschichte APuZ (2006), Parallelgesellschaften? bpb, 1-2/2006. Available online at: <http://www.bpb.de/shop/zeitschriften/apuz/30001/parallelgesellschaften> [Zugriff $07 / 11 / 2018$ ]

Bukow W. et al. (2007), Was heißt hier Parallelgesellschaft? Wiesbaden, VS Verlag für Sozialwissenschaften.

DAмm A.P. (2009), Ethnic Enclaves and Immigrant Labor Market Outcomes: Quasi-Experimental Evidence. In: Journal of Labor Economics 27 (2), pp. 281-314, checked on 11/14/2018.

Der Standard (07/07/2017a), Schweinefleischverbot im Kindergarten: Aufregung um TVBericht - derstandard.at/2000060991866/Schweinefleischverbot-im-Kindergarten-Aufre gung-um-TV-Bericht.Available online at: $<$ https://derstandard.at/2000060991866/Schweine fleischverbot-im-Kindergarten-Aufregung-um-TV-Bericht> [Zugriff 07/11/2018]

DER STANDARD (1/12/2017b), Mehrheit der Österreicher sieht muslimische Parallelgesellschaft. Availableonlineat: $<$ https://derstandard.at/2000068874413/IntegrationsbarometerMehrheitsieht-muslimische-Parallelgesellschaft $>$ [Zugriff 07/11/2018]

Eickelman D. (2002), The Middle East and Central Asia. An Anthropological Approach. New Jersey: Prentice Hall.

Esser H. (1990), Interethnische Freundschaften. In: Esser H. \& Friedrichs, J. (eds.): Generation und Identität. Theoretische und empirische Beiträge zur Migrationssoziologie. Wiesbaden, Vieweg+Teubner Verlag (Studien zur Sozialwissenschaft, 97), pp. 185-205.

Esser H. (2001), Integration und ethnische Schichtung. Arbeitspapier Nr. 40 des Mannheimer Zentrums für europäische Sozialforschung. Mannheim.

FAssin D. (2005), Compassion and Repression. The Moral Economy of Immigration Policies in France. In Cultural Anthropology 20 (3), pp. 362-387.

Fischer, C.S. (1982), To dwell among friends: Personal networks in town and city. University of Chicago Press.

Forrest R., \& KeARns A. (2001), Social cohesion, social capital and the neighbourhood. Urban Studies, 38 (12), pp. 2125--2143.

Forschungsbereich beim SachVerständigenrat Deutscher Stiftungen für Intgration und Migration (2017), Wie gelingt Integration? Asylsuchende über ihre Lebenslagen und Teilhabeperspektiven in Deutschland. Herausgegeben vom SVR-Forschungsbereich und der Robert Bosch Stiftung. Berlin.

Gaitanides S. (2001), Die Legende der Bildung von Parallelgesellschaften. In: iza-Zeitschrift für Migration und soziale Arbeit, 3 (4), pp. 16-25. 
Gemeinschaftswerk der Evangelischen Publizistik GEP (ed.) (2005), Leben Migrantinnen und Migranten in ,parallelen Welten“? Möglichkeiten der Integration. Frankfurt am Main, Tagungsdokumentation Nr. 37.

Halm D. \& SAUER M. (2006), Parallelgesellschaft und ethnische Schichtung. In: Aus Politik und Zeitgeschichte, Berlin.

HeITMEYER W. (1996), Für türkische Jugendliche in Deutschland spielt der Islam eine wichtige Rolle: Erste empirische Studie: 27 Prozent befürworten Gewalt zur Durchsetzung religiöser Ziele. In: DiE ZeIt, Archiv. Available Online at: <https://www.zeit.de/1996/35/heitmey. txt.19960823.xml/komplettansicht> [Zugriff 16/01/2019]

HeItMeyer W. et al. (1997), Verlockender Fundamentalismus. Türkische Jugendliche in Deutschland. Frankfurt am Main, Suhrkamp (Edition Suhrkamp Kultur und Konflikt).

HeItMEYER W. et al. (eds.) (1998), Die Krise der Städte: Analyse zu den Folgen desintegrativer Stadtentwicklungen für das ethnisch-kulturelle Zusammenleben. Frankfurt/Main, Suhrkamp.

Janssen A. \& Polat A. (2006), Soziale Netzwerke türkischer Migrantinnen und Migranten. In: Bundeszentrale für politische Bildung bpb (ed.), Parallelgesellschaften? Aus Politik und Zeitgeschichte APuZ (1-2), pp. 11-17.

Kamal N. (2019), Neighborhood in Nablus City (Working title). (Unpublished).

Karpenstein J. et al. (2007), Abschließender Projektbericht: Integration und Politik in der multikulturellen Stadt. Humboldt Universität Berlin. Available online at: $<$ https://www. sowi.hu-berlin.de/de/lehrbereiche/stadtsoz/forschung/al/Abschlussbericht $\% 20 \mathrm{PSE} \% 20$ Multikulti.pdf $>$ [Zugriff 15/01/2019]

Kazim H. (24/12/2010), Sarrazins Sündenböcke. Edited by SpIegel. Available online at: <http:// www.spiegel.de/politik/deutschland/integrationsdebatte-sarrazins-suendenboecke-a735774.html> [Zugriff 07/11/2018]

Khawaja N.G. et al. (2008), Difficulties and coping strategies of Sudanese refugees: a qualitative approach. In: Transcultural Psychiatry 45 (3), pp. 489-512.

Khvorostianov N. et al. (2012), 'Without it I am nothing': The internet in the lives of older immigrants. In New Media \& Society 14 (4), pp. 583-599.

KROHN K. (24/07/2018), Interview mit Integrationsforscher Haci-Halil Uslucan „Özil ist nicht das richtige Beispiel ". Edited by Stuttgarter Zeitung. Available online at: $<$ https://www. stuttgarter-nachrichten.de/inhalt.interview-mit-integrationsforscher-oezil-ist-nicht-dasrichtige-beispiel.46a6672e-c8e9-476f-9573-03ee398f7fae.html> Zugriff 07/11/2018.

KÜCÜK E. \& FisCHER K. (02/09/2018), „Für die Errungenschaften der offenen Gesellschaft kämpfen". Deutschlandfunk (ed.), Available online at: <https://www.deutschlandfunk. de/politologin-esra-kuecuek-fuer-die-errungenschaften-der.911.de.html?dram:article_ id $=427038>$ [Zugriff 31/01/2019]

Kurz S. (21/10/2018), Parallelgesellschaften verhindern. Available online at: <https://www. sebastian-kurz.at/programm/>

Lin C.A. \& AtKin D. (eds.) (2002), Communication technology and society: Audience adoption and uses. Cresskill, NJ, Hampton Press.

Linnert J. \& Berg T. (2016), Freiwilligenarbeit mit Geflüchteten. Forschungsstand zum freiwilligen Engagement mit Geflüchteten unter Aspekten gesellschaftlicher Teilhabe und politischer Bildung. Working Paper I des Projekts Perspektive. Gefördert von der Robert Bosch Stiftung. Berlin.

Lockwood D. (1964), Social Integration and System Integration. In: Zollschan G. \& HiRsch W. (eds.), Explorations in Social Change, London, Houghton Mifflin. 
Long N.J. \& Moore H.L. (2013), Introduction: sociality's new directions. In: Long N.J. \& Moore H.L. (eds.), Sociality. New directions. New York, Berghahn (Wyse Series in Social Anthropology, 1), pp. 1-29.

Long N.J. \& Moore H.L. (eds.) (2013), Sociality. New directions. New York, Berghahn (Wyse Series in Social Anthropology, 1).

Malik S.H. \& Coulson N.S. (2010), Coping with infertility online: an examination of self-help mechanisms in an online infertility support group. In: Patient Education and Counseling 81 (2), pp. 315-318.

Manea E. (2017), „Geschlossene Gesellschaften weisen meist patriarchalische Machtstrukturen auf, es herrscht eine starke soziale Kontrolle.“ In: ÖsterReICHISCHER InTEGRATIONSFONDS (ed.), Parallelgesellschaften. Segregation und desintegrative Milieus. Vienna (Perspektiven Integration), pp. 6-11.

Meyer T. (2002), Parallelgesellschaft und Demokratie. In: Meyer T. \& Weil R. (eds.), Die Bürgergesellschaft. Perspektiven für Bürgerbeteiligung und Bürgerkommunikation. Friedrich-Ebert-Stiftung. Bonn, Dietz, pp. 343-372.

Meyer T. \& WeIL R. (eds.) (2002), Die Bürgergesellschaft. Perspektiven für Bürgerbeteiligung und Bürgerkommunikation. Friedrich-Ebert-Stiftung. Bonn, Dietz.

MiKal J.P. et al. (2013), Transition, stress and computer-mediated social support. In Computers in Human Behavior 29 (5), A40-A53.

MiKal J.P., \& Woodfield B. (2015), Refugees, post-migration stress, and internet use: a qualitative analysis of intercultural adjustment and internet use among Iraqi and Sudanese refugees to the United States. Qualitative Health Research, 25 (10), 1319-1333.

Newman J. \& Tonkens E. (2011), Participation, responsibility and choice: summoning the active citizen in western European welfare states. Amsterdam, University Press.

Österreichischer Integrationsfonds (2017), Parallelgesellschaften. Segregation und desintegrative Milieus. Wien (Perspesktiven Integration). Available online at: $<$ https://www.integra tionsfonds.at/fileadmin/content/AT/Fotos/Logos_Sujets/Allgemeine/3_Perspektiven_ Parallelgesellschaft_200x260_SCREEN.pdf $>$ [Zugriff 31/01/2019]

OnyUt L.P. et al. (2009), Trauma, poverty and mental health among Somali and Rwandese refugees living in an African refugee settlement - an epidemiological study. In Conflict and Health 3, p. 6. Available online at: <https://conflictandhealth.biomedcentral.com/ articles/10.1186/1752-1505-3-6> [Zugriff 10/02/2019]

Rains S.A. \& Young V. (2009), A Meta-Analysis of Research on Formal Computer-Mediated Support Groups: Examining Group Characteristics and Health Outcomes. Human Communication Research 35 (3), pp. 309-336.

Sangalang C. et al. (2018), Trauma, Post-Migration Stress, and Mental Health: A Comparative Analysis of Refugees and Immigrants in the United States. In: Journal of Immigrant and Minority Health, available online: <doi: 10.1007/s10903-018-0826-2> [Epub ahead of print].

SCHIFfaUer W. (2005), Parallelgesellschaften“ und islamische Gemeinden. In: GemeInSCHAFTSWERK der Evangelischen Publizistik GEP(ed.): Leben Migrantinnen und Migranten in , parallelen Welten"? Möglichkeiten der Integration. Frankfurt am Main, Tagungsdokumentation Nr. 37, pp. 20-28.

Schuleri-Hartje U. (1982), Ausländische Arbeitnehmer und ihre Familien. Teil 1: Wohnverhältnisse. Berlin. (Deutsches Institut für Urbanistik). 
SinNERBRINK I. et al. (1997), Compounding of premigration trauma and postmigration stress in asylum seekers. In The Journal of Psychology 131 (5), pp. 463-470.

StaAs C. (10/02/2011), Neuer Streit, altes Lied. Warum die Deutschen alle Jahre wieder über Einwanderung und Integration debattieren. Ein Gespräch mit dem Historiker Ulrich Herbert. ZEIT (ed.). Available online at: <https://www.zeit.de/2011/07/U-Herbert-Einwanderung > [Zugriff 07/11/2018]

Thomése F., \& VAN TilbuRg T. (2000), Neighbouring networks and environmental dependency. Differential effects of neighbourhood characteristics on the relative size and composition of neighbouring networks of older adults in The Netherlands. Ageing \& Society, 20 (1), pp. $55-78$.

ToReN C. (2013), Imagining the World that Warrants Our Imagination. The Revelation of Ontogeny. In: Long N.J \& Moore, H.L. (eds.), Sociality. New directions. New York, Berghahn (Wyse Series in Social Anthropology, 1), pp. 43-59.

Turner V. (1995), Vom Ritual zum Theater. Der Ernst des menschlichen Spiels. Ungek. Ausg. Frankfurt am Main, Fischer-Taschenbuch-Verl. ([Fischer-Taschenbücher], 12779).

Ulram P. (2009), Integration in Österreich. Einstellungen, Orientierungen, Erfahrungen von MigrantInnen und Angehörigen der Mehrheitsbevölkerung. GfK Austria GmbH Wien.

VAn den Berg P., \& Timmermans H. (2015), A multilevel path analysis of social networks and social interaction in the neighbourhood. Region, 2 (1), pp. 55-66.

Van der Poel M.G. (1993), Delineating personal support networks. Social Networks, 15 (1), pp. 49-70.

Walther J.B. \& Boyd S. (2002), Attraction to computer-mediated social support. Available online at: $<$ https://msu.edu/walther/docs/support.html $>$ Reprint In: Lin C.A. \& ATKIN D. (eds.), Communication technology and society: Audience adoption and uses. Cresskill, NJ, Hampton Press, pp. 153-188.

WeHr H. (1985), Arabisches Wörterbuch für die Schriftsprache der Gegenwart (5. Auflage). Harrassowitz Verlag, Wiesbaden.

Wolf F. (2017), Vorwort. In ÖsterReichischer InTEGRATIONSFOnds (eds.), Parallelgesellschaften. Segregation und desintegrative Milieus. Vienna (Perspektiven Integration), pp. 4-5. 